\title{
Autism-linked mutations of CTTNBP2 reduce social interaction and impair dendritic spine formation via diverse mechanisms
}

\author{
Pu-Yun Shih ${ }^{1,2}$, Bing-Yuan Hsieh ${ }^{2}$, Ching-Yen Tsai ${ }^{2}$, Chiu-An Lo ${ }^{3}$, Brian E. Chen ${ }^{3,4}$ and Yi-Ping Hsueh , $^{1 *}$ (D)
}

\begin{abstract}
Abnormal synaptic formation and signaling is one of the key molecular features of autism spectrum disorders (ASD). Cortactin binding protein 2 (CTTNBP2), an ASD-linked gene, is known to regulate the subcellular distribution of synaptic proteins, such as cortactin, thereby controlling dendritic spine formation and maintenance. However, it remains unclear how ASD-linked mutations of CTTNBP2 influence its function. Here, using cultured hippocampal neurons and knockin mouse models, we screen seven ASD-linked mutations in the short form of the Cttnbp2 gene and identify that M120I, R533* and D570Y mutations impair CTTNBP2 protein-protein interactions via divergent mechanisms to reduce dendritic spine density in neurons. R533* mutation impairs CTTNBP2 interaction with cortactin due to lack of the C-terminal proline-rich domain. Through an $\mathrm{N}-\mathrm{C}$ terminal interaction, $\mathrm{M} 120 \mathrm{l}$ mutation at the $\mathrm{N}$-terminal region of CTTNBP2 also negatively influences cortactin interaction. D570Y mutation increases the association of CTTNBP2 with microtubule, resulting in a dendritic localization of CTTNBP2, consequently reducing the distribution of CTTNBP2 in dendritic spines and impairing the synaptic function of CTTNBP2. Finally, we generated heterozygous M120l knockin mice to mimic the genetic variation of patients and found they exhibit reduced social interaction. Our study elucidates that different ASD-linked mutations of CTTNBP2 result in diverse molecular deficits, but all have the similar consequence of synaptic impairment.
\end{abstract}

Keywords: Autism spectrum disorder, Cortactin, Dendritic spine formation, F-actin, Microtubule

\section{Introduction}

Autism spectrum disorders (ASD) are highly prevalent neurodevelopmental diseases mainly caused by genetic variations $[2,19]$. Hundreds of mutated genes have been associated with ASD (https://gene.sfari.org/database/ human-gene/). One critical feature of ASD-causative or -linked genes is their roles in controlling synapse

\footnotetext{
*Correspondence: yph@gate.sinica.edu.tw

${ }^{1}$ Molecular and Cell Biology, Taiwan International Graduate Program, Institute of Molecular Biology, Academia Sinica and Graduate Institute of Life Sciences, National Defense Medical Center, Taipei, Taiwan, ROC

Full list of author information is available at the end of the article
}

formation and synaptic signaling and activity [7, 11, 18], suggesting a crucial aspect of synaptic function in ASD.

Among the various synapse-associated ASD candidate genes, CTTNBP2 was identified as a strong candidate ASD-linked gene based on several human genetic studies $[7,11,17,18]$. A study of Cttnbp 2 knockout mice has also indicated that CTTNBP2 regulates social interaction, ultrasonic vocalization and hyperactivity [20], further strengthening the role of CTTNBP2 in ASD. To date, 38 mutations in the CTTNBP2 gene have been identified in patients with ASD (https://gene.sfari.org/database/ human-gene/CTTNBP2\#variants-tab). However, how ASD-linked mutations of CTTNBP2 alter neuronal function to result in autism-like symptoms remains elusive. 


\begin{abstract}
(See figure on next page.)
Fig. $1 \mathrm{M} 1201$ and R533* mutants reduce dendritic spine density and mEPSC frequency. a Upper: Schematic of the protein structure of CTTNBP2. Seven human ASD-linked mutations (in parentheses) and their corresponding residues in mouse protein are indicated. Binding partners of each domain are noted below. N, N-terminal region; CC, coiled-coil domain; Mid, middle region; P-rich, proline-rich domain. Middle: Predicted secondary structure of CTTNBP2 by I-TASSER (https://zhanglab.ccmb.med.umich.edu/I-TASSER). Red part represents alpha-helix, and black represents coil. Lower: IUpred2A (https://iupred2a.elte.hu) prediction of disordered regions of CTTNBP2. Higher score indicates a higher degree of disorder. b Confirmation of expression of Myc-tagged CTTNBP2 ASD-linked mutant proteins in COS1 cells. $\beta$-actin and a-tubulin were used as internal controls. c The effect of CTTNBP2 ASD-linked mutations on dendritic spine density. M120l and R533* mutation showed the most dramatic reduction in dendritic spine density. Representative image of cultured hippocampal neurons that express HA-tagged WT or ASD-linked mutant proteins (viewed in red). Dendritic morphology was outlined by GFP-actin (visualized in green). Quantification results of dendritic spine density are shown. The quantification results of vector, WT and R533* have been reported previously [20]. Since those three groups of experiments were performed at the same time as the experiments on the other mutant proteins targeted for this study, we compare all mutants together here. $\mathbf{d}$ M120l and R533* mutants reduce $m E P S C$ frequency but not amplitude. Data represent mean \pm SEM. Each dot in (c) and (d) indicates the individual result for a dendritic segment and neuron, respectively. The results were collected from three (c) and ten (d) independent cultures. c Kruskal-Wallis test with Dunn's multiple comparison test were used to compare mutant protein with the wild-type (WT) group (*) or vector control (\#). d One-way ANOVA was used for analysis. See Additional file 2: Table S1 for all statistical data and exact sample size for each group. ${ }^{*}$ or $\# P<0.05$; ${ }^{* *} P<0.01$; ${ }^{* * *}$ or \#\#P $<0.001$. Scale bar, $5 \mu \mathrm{m}$
\end{abstract}

Sequence analysis predicted that alternative RNA splicing results in three CTTNBP2 isoforms, i.e. short, long and intron forms, but the protein products of the long and intron forms of CTTNBP2 are not detectable in the brain $[5,14]$. Instead, a protein species of $\sim 90 \mathrm{kDa}$, i.e. the short form, is the only detectable protein product of CTTNBP2 in the brain $[5,14,20,21]$. Therefore, mutations within the CTTNBP2 short form, hereafter simply denoted CTTNBP2, are expected to be more relevant to ASD, so we have focused on seven such ASD-linked mutations in this report.

CTTNBP2 consists of an N-terminal coiled-coil domain for homo- and hetero-oligomerization, a middle region for microtubule binding, and a C-terminal proline-rich domain that interacts with cortactin (Fig. 1a) $[4,14,21]$. CTTNBP2 modulates neuronal morphogenesis by regulating actin and microtubule dynamics [9]. In immature neurons, CTTNBP2 associates with microtubule and promotes microtubule stability along dendrites. This stabilization contributes to dendritic arborization [21]. As neurons mature, CTTNBP2 shifts from the dendritic shaft into dendritic spines where it facilitates synaptic targeting of cortactin [5]. This role in synaptic targeting is critical for dendritic spine formation and maintenance because both Cttnbp 2 knockdown and expression of a CTTNBP2 mutant that cannot interact with cortactin results in reduced dendritic spine density and size [5]. Apart from its contribution to cytoskeleton dynamics, proteomic analysis has further indicated that CTTNBP2 controls the synaptic expression of more than a hundred proteins, including SHANK2, SHANK3, striatin (STRN), and RAC3 [20]. These synaptic proteins may also contribute to the function of CTTNBP2 in neurons.

In this study, we utilized cultured neurons and knockin mice carrying ASD-linked mutations in CTTNBP2 to investigate how these mutations alter
CTTNBP2 activity and influence neuronal morphology and function. We found that although different mutations impact divergent molecular functions of CTTNBP2, they all modulate dendritic spine density. Our study sheds light on the multiple regulatory roles of CTTNBP2 in dendritic spine formation and provides mechanistic evidence for how CTTNBP2 mutations contribute to ASD etiology.

\section{Materials and methods Experimental design}

Since seven ASD-linked mutations in human CTTNBP2 are conserved among human, rat and mouse (Additional file 1: Fig. S1), we used rat cultured hippocampal neurons and knockin mice to analyze how ASD-linked mutations influence the function of CTTNBP2 in terms of dendritic spine density, neuronal activity and mouse behaviors. We primarily overexpressed ASD-linked mutations in wild-type (WT) hippocampal neurons and used heterozygous knockin mice to mimic monoallelic mutations of CTTNBP2 in patients. Based on our analysis of dendritic spine formation in cultured neurons, we then focused on three mutations, i.e. M120I, R533* and D570Y, because they altered the subcellular distribution of CTTNBP2 and impaired dendritic spine formation. A series of biochemical and cell biology approaches were employed to dissect the molecular defects caused by ASD mutations. Neuronal morphology and mouse behaviors of ASD mutant mice were then investigated to elucidate the physiological impact of ASD-linked mutations in the Cttnbp 2 gene in vivo. All morphometric, electrophysiological and behavioral analyses were conducted blind by relabeling the samples before analysis by another member in the laboratory. All statistical methods and results are summarized in Additional file 2: Table S1, Additional file 3: Table S2. 


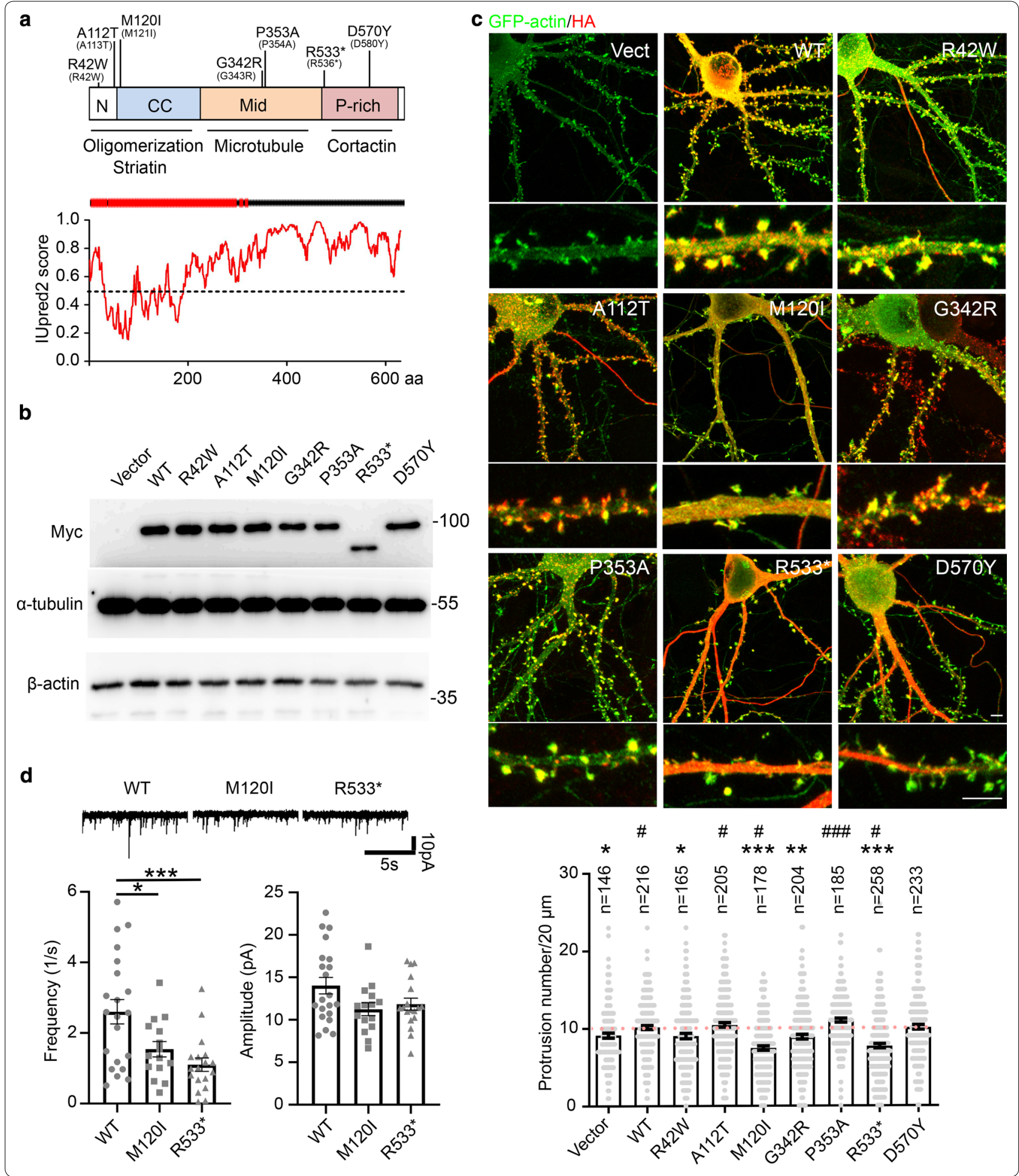

Animals

All the animal experiments in this study were performed with the approval of the Academia Sinica Institutional Animal Care and Utilization Committee (Protocol \#
12-10-414 and 11-12-294). Animal housing and handling were conducted according to the guidelines of the Council of Agriculture Guidebook for the Care and Use of Laboratory Animals. For primary hippocampal neuron 
culture, pregnant rats were sacrificed by $\mathrm{CO}_{2}$ inhalation. Embryonic day E18.5 fetal pups of both sexes were isolated and sacrificed by decapitation. Male mice at the age of 2-3 months were used for behavioral assays. All animals were housed and bred in the animal facility of the Institute of Molecular Biology, Academia Sinica, under controlled humidity and temperature and a $12 \mathrm{~h}$ light/ dark cycle (light off at 20:00). Animals accessed water and food (\#5K54, LabDiet) ad libitum. All genetically modified mice had been backcrossed to WT C57BL/6 mice for more than six generations to minimize off-target effects of CRISPR/Cas9 editing.

\section{CRISPR/Cas9 technology for introducing ASD-associated mutations into Cttnbp2}

A paired-nicking approach $[22,23]$ was applied to insert mutation sequences into exon 4 of Cttnbp2. For the Cttnbp2 M120I mutation, the paired guide RNAs (5'AGAAAGGATGTCCGCACAGC and 5'- AGTGGG CCATGACAGCTTCA) and a single-stranded DNA template (ssODN: 5'- TGTGAGCCAGTTCTGCTGTTC CTTGCTATTATGGGAAAAACAGATGACGTCTCA GTACCTTTTTTTGTCTGCTCTCAGCGGCCACCA GCTGTGCGGACATCCTTTCTTGtATTTTTCTaCAGTGGGCCATGACAGCTTCAAGGATGGAGAGT GGATTGGTGCAGACTGGCTTCTCTTTGTCACCA GGACCTGCTTCATAG) were used. Two nucleotides (in lower case) were changed in order to alter the coding sequences of methionine to isoleucine at residue 120 (shown in bold and underlined) and to disrupt the PstI site (underlined) without changing the encoded amino acid.

For the Cttnbp2 D570Y mutation, a guide RNA (5'ATCAACTTTGGCCCCTGCAT) and a single-stranded DNA template (ssODN: 5'- GGAAATCCTCCTCCTATC CCTCCCAAAAAGCCAGGGCTCTCCCAAACTCCT TCTCCGCCACACCCCCAACTGAGGGCCTCCAAT GCAGGGGCCAAAGTTtATAACAAgATTGTGGCT TCACCTCCCTCTACTTTGCCACAAGG) were used. Two nucleotides (lower case) were changed in order to alter the coding sequence from aspartic acid to tyrosine at residue 570, and to create a Psil site and disrupt the MluCI site without changing the encoded amino acid, respectively.

A T7 promoter sequence (5'- TTAATACGACTC ACTATA) was added upstream of gRNA sequences and a partial tracrRNA sequence (5'- GTTTTAGAG CTAGAAATAGC) was added downstream of the gRNA sequence. The oligo was annealed with reverse tracrRNA (5'-TTTAAAAGCACCGACTCGGTG CCACT T T T T CAA GT TGATAACGGACTAGCC TTATTTTAACTTGCTATTTCTAGCTCTAAAAC) and PCR-amplified using Phusion DNA Polymerase (Thermo-Fisher Scientific) according to the manufacturer's instructions. The amplified product was purified using QIAquick PCR Purification Kit (28106, Qiagen) and it served as the in vitro transcription template.

The sgRNAs were synthesized using HiScribe ${ }^{\text {TM }}$ T7 Quick High Yield RNA Synthesis Kit from NEB (2050S, NEB). Cas9A ${ }^{\text {D10A }}$ mRNA was synthesized using mMESSAGE mMACHINE T7 Ultra Kit (AM1345, ThermoFisher Scientific) using pCAG-T3-hCasD10A-pA (\#51638, Addgene) as template, purified using MEGAclear Transcription Clean-up Kit (AM1908, ThermoFisher Scientific), and eluted with injection buffer (10 mM Tris- $\mathrm{HCl}$ pH7.2 and $0.1 \mathrm{mM}$ ethylenediaminetetraacetic acid (EDTA)). The quality and quantity of RNAs were analyzed using a NanoDrop ND-1000 (Thermo-Fisher Scientific). To extract genomic DNA, $\sim 3 \mathrm{~mm}$ of mouse tail was cut and lysed in $600 \mu \mathrm{l}$ $50 \mathrm{mM} \mathrm{NaOH}$ and boiled for $30 \mathrm{~min}$. After complete tissue lysis, samples were cooled down briefly on ice before adding $60 \mu \mathrm{l}$ of $1 \mathrm{M}$ Tris- $\mathrm{Cl} \mathrm{pH} 8.0$ to neutralize the $\mathrm{pH}$. Around 0.5-1 $\mu \mathrm{l}$ of DNA was used for PCR reaction. Primer sequences to amplify the M120I mutant allele by PCR were $5^{\prime}$-ATGGCTTTCCAGGCT TGTCAG- $3^{\prime}$ and $5^{\prime}$-AGCCCACTCCCACCAAAA CTA-3'. To amplify the D570Y mutant allele by PCR, we designed the primer set: 5'-GCCAAGCAGCTA GCTCGGAATAC- $3^{\prime}$ and $5^{\prime}$-GTTCAGTCCAGGGGT TCCAGCAG- $3^{\prime}$. To distinguish M120I and D570Y alleles, we digested the PCR product by PstI (NEB) and $M l u C I$ (NEB), respectively.

\section{Plasmids}

For immunostaining and biochemical analysis, the GW1HA-CTTNBP2, GW1-myc-CTTNBP2, GW1-myc-NCC, and GW1-myc-Mid constructs were described previously $[4,5,21]$. For mEPSC recording (Fig. 1d), pCAGGFP-P2A was constructed by inserting SuperfolderGFP and $P 2 A$ sequences into an empty pCAG vector [13]. The original superfolder GFP was obtained from [15], the P2A sequence is from [13], and the empty pCAG vector was ordered from Addgene. HA-tagged Cttnbp 2 was PCR-amplified using the primer set $5^{\prime}$ - GCGATATCT TAGGGAGGGTG- $3^{\prime}$ and $5^{\prime}$ - CCAGATATCATGTAC CCATATGAC $-3^{\prime}$ and cloned into pCAG-GFP-2A vector plasmid at the EcoRV site. Myc-cortactin was a generous gift from Dr. Morgan Sheng at MIT. To construct the GFP-P-rich domain, the P-rich domain was PCR-amplified using the primer set $5^{\prime}$-GGAATTCCGAACCGG TTTAAAG- ${ }^{\prime}$ and $5^{\prime}$-GGAATTCTTAGGGAGGGTG- ${ }^{\prime}$ and cloned into pEGFP-C2 plasmid at the EcoRI site. Site-directed mutagenesis using PCR was performed to 
construct ASD-associated Cttnbp 2 mutant constructs. Sequences of all primer pairs (from $5^{\prime}$ to $3^{\prime}$ ) are as follows (uppercase letters indicate mutated residues):

\begin{tabular}{ll}
\hline BP2 R42W-F & cgctcagcaaatcagagctgTggatgctccttagcgtgatg \\
BP2 R42W-R & catcacgctaaggagcatccAcagctctgatttgctgagcg \\
BP2 A112T-F & ccactctccatccttgaaActgtcatggcccactgag \\
BP2 A112T-R & ctcagtgggccatgacagTttcaaggatggagagtgg \\
BP2 M120I-F & catggcccactgcagaaaaatAcaagaaaggatgtccgcac \\
BP2 M120l-R & gtgcggacatcctttcttgTatttttctgcagtgggccatg \\
BP2 G342R-F & tagttcccacaaacacaaaaAggaatgtgggccccagtgcc \\
BP2 G342R-R & ggcactggggcccacattccTttttgtgtttgtgggaacta \\
BP2 P353A-F & ccagtgccctgctgattagaGcaggtattgataggcagtct \\
BP2 P353A-R & agactgcctatcaatacctgCtctaatcagcagggcactgg \\
BP2 R533X-F & taaagactcccggggcagcaTgagttgacagaggaaatcctcc \\
BP2 R533X-R & ggaggatttcctctgtcaactcAtgctgccccgggagtcttta \\
BP2 D570Y-F & ccaatgcaggggccaaagttTataacaaaattgtggcttc \\
BP2 D570Y-R & gaagccacaattttgttatAaactttggcccctgcattgg \\
\hline
\end{tabular}

\section{Antibodies}

The following antibodies and working concentration were used in this study: anti-CTTNBP2 (A5, A7, and 9W, rabbit, homemade, $0.5 \mu \mathrm{g} / \mathrm{ml}$ ) [5, 20], anti-Myc tag (9B11, Cell Signaling Technology, 1/1000 for staining; 06-549, Millipore, $1 \mu \mathrm{g} / \mathrm{ml}$ ), anti-HA tag (3F10, Roche, $0.5 \mu \mathrm{g} / \mathrm{ml}$ ), anti-HA tag (Y-11, Santa Cruz Biotechnology, $0.5 \mu \mathrm{g} / \mathrm{ml}$ ), anti-GFP (ab13970, Abcam, $0.5 \mu \mathrm{g} / \mathrm{ml}$ ), antiatubulin (B-5-1-2, Sigma-Aldrich, $1 \mu \mathrm{g} / \mathrm{ml}$ ), anti-acetyl tubulin (6-11B-1, Sigma-Aldrich, $1 \mu \mathrm{g} / \mathrm{ml})$, anti-ßactin (AC-74, Sigma-Aldrich, 1/1000), anti-cortactin (H-191, Santa Cruz Biotechnology, $0.5 \mu \mathrm{g} / \mathrm{ml}$ ), anti-FOS (\#2250, clone 9F6, Cell Signaling Technology, 1/200), anti-mouse HRP (NA931, GE Healthcare, 1/5000), anti-rabbit HRP (NA934, GE Healthcare, 1/5000), anti-chicken Alexa Fluor 488 (A-11039, Invitrogen, $1 \mu \mathrm{g} / \mathrm{ml}$ ), anti-mouse Alexa Fluor 555 (A-21424, Invitrogen, $1 \mu \mathrm{g} / \mathrm{ml}$ ), anti-rat Alexa Fluor 594 (A-21209, Invitrogen, $1 \mu \mathrm{g} / \mathrm{ml}$ ), and antirabbit Alexa Fluor 647 (A-21244, Invitrogen, 2 rg/ml).

\section{Preparation and transfection of cultured primary hippocampal neurons}

Preparation of primary rat hippocampal culture was described previously $[5,21]$. Briefly, hippocampi were carefully collected at embryonic day E18.5 and digested with papain solution $[0.6 \mathrm{mg} / \mathrm{ml}$ papain, $0.5 \mathrm{mM}$ EDTA, $1.5 \mathrm{mM} \mathrm{CaCl}, 0.06 \%$ DNase I, $0.2 \mathrm{mg} / \mathrm{ml}$ cysteine] at $37^{\circ} \mathrm{C}$ for $25 \mathrm{~min}$. The papain solution was removed and the digested hippocampi were gently washed with Hank's balanced salt solution (HBSS) buffer. To dissociate the cells, the digested hippocampi were gently pipetted. The cell suspension without debris was then transferred to a new tube and centrifuged at $900 \mathrm{rpm}$ for $5 \mathrm{~min}$ to collect dissociated cells. The cell pellets were re-suspended and cell density was determined. For a 12-well plate, $2 \times$ $10^{5}$ cells/well were seeded on a polylysine-coated glass coverslip.

\section{Cresyl violet staining}

Fifty- $\mu$ m-thick brain sections were mounted onto glass slides coated with $0.5 \%$ gelatin and air-dried. The sections were then stained with cresyl violet solution $(0.1 \%$ cresyl violet in $1 \%$ acetic acid) and destained several times with $70 \%$ ethanol until the signal was clear. The sections were serially dehydrated with 70\%, $90 \%$ and $100 \%$ ethanol and then xylene for mounting using Permount mounting medium (Fisher Scientific).

\section{Immunostaining}

For neuron morphology analysis, rat primary cultured hippocampal neurons were fixed by $4 \%$ paraformaldehyde with $4 \%$ sucrose in phosphate-buffered saline (PBS) for $10 \mathrm{~min}$ and permeabilized by $0.2 \%$ Triton $\mathrm{X}-100$ in PBS for $10 \mathrm{~min}$. After washing with PBS, the sections were blocked with $10 \%$ Bovine serum albumin (BSA) for $30 \mathrm{~min}$ in room temperature, and incubated with primary antibodies in $3 \%$ BSA overnight in $4{ }^{\circ} \mathrm{C}$. After washing with PBS, the coverslips were incubated with secondary antibodies $3 \%$ BSA for $1 \mathrm{~h}$ in room temperature. For C-FOS staining, two hours after the reciprocal social stimulation test, the brain was fixed with $4 \%$ paraformaldehyde in PBS. Fifty $\mu \mathrm{m}$-thick brain sections were treated with $1 \% \mathrm{H}_{2} \mathrm{O}_{2}$ in Tris-Cl buffer, $\mathrm{pH}$ 7.6, for $30 \mathrm{~min}$ and permeabilized with $0.05 \%$ Tween-20 in PBS for $15 \mathrm{~min}$. After washing with PBS, the sections were blocked with TNB buffer $(0.5 \%$ blocking reagent in PBS, TSA Fluorescein System Kit, No.1715186, Perkin Elmer) for $1 \mathrm{~h}$ and then incubated overnight with primary antibody in TNB buffer at $4{ }^{\circ} \mathrm{C}$. After washing with $0.05 \%$ Tween-20 in PBS, sections were incubated with biotinylated goat antirabbit IgG secondary antibody $(1 / 200$, vectastain, Vector Laboratories) in TNB buffer for $2 \mathrm{~h}$. The immunoreactivity was developed using Vectastain Elite ABC Kit (Vector Laboratories) based on the manufacturer's instructions. For immunofluorescence staining, brain sections were incubated with primary antibody as described above, followed by incubation with the secondary antibodies conjugated with Alexa Fluor-488, -555, -594, and/or -647 (Invitrogen) for $2 \mathrm{~h}$.

\section{Microscopy and morphometry analyses}

True-color imaging (for C-FOS staining and Cresyl violet stain) was performed using an upright microscope (Microscope Axio Imager M2, Carl Zeiss) equipped 
with a $10 \times /$ NA 1.4 oil (Plan-Apochromat, Carl Zeiss) objective lens, and AxioCam (Carl Zeiss) and Zen 2011 software (Carl Zeiss). Shading correction and white balance was applied to correct the signal. For cresyl violet staining, the images were tiled up to acquire entire sections. Fluorescence images were captured using a confocal microscope (LSM700, Carl Zeiss) equipped with a $63 \times /$ NA 1.4 oil objective lens (Plan-Apochromat, Carl Zeiss) and Zen 2009 (Carl Zeiss) at room temperature. To analyze spine morphology of hippocampal CA1 in vivo, Cttnbp 2 mutant mice were crossed with Thy1-YFP transgenic mice (\#003782, Thy1-YFP-H, The Jackson Laboratory) [8]. The first branch of the apical dendrite of CA1 pyramidal neurons was selected for analysis. A $15-\mu \mathrm{m}$-long dendritic fragment $5 \mu \mathrm{m}$ distant from the branch point was used to determine the density and length of spines and the width of spine heads. The Z-series images were captured at $0.2 \mu \mathrm{m}$ intervals with the "Region" function in Zen 2009 (Carl Zeiss) and processed using the "maximum projection" function. Quantifications were performed using ImageJ. The spine density of two or three dendritic segments from the same neuron was averaged to represent the density of each neuron. Ten neurons were imaged from each animal and at least three mice were used for each group of an experiment.

For cultured hippocampal neurons, we focused on a $20-\mu \mathrm{m}$-long segment of the primary dendrite starting $20 \mu \mathrm{m}$ away from the soma to measure dendritic spine density and the synaptic distribution of CTTNBP2 and cortactin. At least two clearly recognized dendrites were quantified and averaged (using blinded sample relabeling) for each neuron to represent the spine density or protein distribution of each neuron. The sample sizes of examined neurons and dendritic segments are summarized in Additional file 2: Table S1. To quantify the synaptic enrichment of cortactin, we quantified the cortactin signal in individual spines and normalized them with the average signal of cortactin in the soma of the same neuron. To quantify synaptic CTTNBP2, we performed line scanning from the tip of the dendritic spine to the dendritic shaft. The CTTNBP2 signals along the lines were determined using the line scanning method in ImageJ $(\mathrm{NIH})$. The signal within the range of $0-0.5 \mu \mathrm{m}$ from the spine tip indicated the synaptic region, whereas signals within the range 1-1.5 $\mu \mathrm{m}$ from the tip represented the base of the dendritic spine and the dendritic shaft. Five spines of each neuron were randomly sampled blindly from thirty-five neurons for each group.

To quantify the acetyl-tubulin/tubulin ratio in neurons, we measured a $5-\mu \mathrm{m}$ segment of primary dendrite within the range of 5-20 $\mu \mathrm{m}$ away from the soma, and used the $\alpha$-tubulin signal to normalize the acetyl-tubulin signal.
This ratio represents microtubule stability in proximal dendrites.

Detailed information on morphometry sampling (number of examined mice, neurons, dendrites, and spines) is presented in Additional file 2: Table S1.

\section{mEPSC recording}

Cultured rat hippocampal neurons were transfected at 14 DIV, and whole-cell patch-clamps were performed at 18 DIV to record miniature EPSCs (mEPSCs). Neurons were incubated in extracellular solution containing $136.5 \mathrm{mM} \mathrm{NaCl}, 5.4 \mathrm{mM} \mathrm{KCl}, 1.8 \mathrm{mM} \mathrm{CaCl} \cdot 2 \mathrm{H}_{2} \mathrm{O}$, $0.53 \mathrm{mM} \mathrm{MgCl} \mathrm{m}_{2} \cdot 6 \mathrm{H}_{2} \mathrm{O}, 5.56 \mathrm{mM}$ Glucose, $5 \mathrm{mM}$ HEPES (4-(2-hydroxyethyl)-1-piperazineethanesulfonic acid) $\mathrm{pH} 7.4(\mathrm{NaOH}), 0.001 \mathrm{mM}$ tetrodotoxin, and $0.02 \mathrm{mM}$ bicuculline. The intracellular solution contained $140 \mathrm{mM}$ K-gluconate, $5 \mathrm{mM} \mathrm{NaCl}, 2 \mathrm{mM}$ EGTA (ethylene glycolbis( $\beta$-aminoethyl ether)-N,N, $\mathrm{N}^{\prime}, \mathrm{N}^{\prime}$-tetraacetic acid), $10 \mathrm{mM}$ HEPES, $4 \mathrm{mM}$ Mg-ATP, $0.3 \mathrm{mM} \mathrm{Na-GTP} \mathrm{pH}$ $7.3(\mathrm{KOH})$. Neurons were voltage-clamped at $-70 \mathrm{mV}$, and mEPSCs were recorded with an Axon Axopatch 200B amplifier (Molecular Devices) and filtered at $1 \mathrm{kHz}$. Clampfit software (10.4; Molecular Devices) was used to detect mEPSCs from the raw data with an amplitude threshold of $5 \mathrm{pA}$.

\section{Immunoprecipitation}

The antibody-protein A complex was first prepared by incubating $20 \mu \mathrm{l}$ of myc antibody (9B11, Cell Signaling) with $20 \mu \mathrm{l}$ of Protein A beads (17046901, GE Healthcare) overnight and washing with PBS to remove unbound antibody. To prepare protein extract for cortactin/oligomerization coimmunoprecipitation experiments, COS1 cell lysates were extracted with RIPA buffer [1\% Triton X-100, $0.1 \%$ sodium dodecyl sulfate (SDS), $1 \%$ sodium deoxycholate, $50 \mathrm{mM}$ Tris- $\mathrm{Cl} \mathrm{pH} 7.4,150 \mathrm{mM}$ $\mathrm{NaCl}, 2 \mathrm{mM}$ EDTA and protease inhibitors] and the debris was removed by centrifugation $(16,000 \times g$ for 20 min at $4{ }^{\circ} \mathrm{C}$ using a table-top microcentrifuge, Heraeus Biofuge Fresco). Lysate was incubated with myc tag antibody-coated Protein A beads for $4 \mathrm{~h}$ at $4{ }^{\circ} \mathrm{C}$ and washed once with each of the following buffers: (1) RIPA buffer, (2) $10 \mathrm{mM}$ Tris-Cl, $1 \%$ Triton $\times 100, \mathrm{pH} 7.4,(3)$ $10 \mathrm{mM}$ Tris-Cl, $0.1 \%$ Triton $\times 100,0.5 \mathrm{M} \mathrm{LiCl}, \mathrm{pH} 7.4$, and (4) $10 \mathrm{mM}$ Tris-Cl, pH7.4. For GFP-P-rich coimmunoprecipitation, lysates were extracted with $1 \%$ Triton $\times$ 100 in PBS and incubated with myc tag antibody-coated Protein A beads for $4 \mathrm{~h}$ at $4{ }^{\circ} \mathrm{C}$ and washed three times with PBS. After removing the final wash buffer, $2 \times$ SDSPAGE sample buffer (4\% SDS, $0.2 \%(\mathrm{w} / \mathrm{v})$ bromophenol blue, $20 \%$ (v/v) glycerol, $200 \mathrm{mM} \beta$-mercaptoethanol) was added and boiled for $10 \mathrm{~min}$. 


\section{Microtubule spin down assay}

A Microtubule Binding Protein Spin-down Assay Biochem Kit (BK029, Cytoskeleton, Denver, CO) was used to test the interaction of CTTNBP2 with microtubule according to the manufacturer's instructions. Tubulin solution was added into General Tubulin Buffer with Taxol (GTB; $80 \mathrm{mM}$ PIPES pH 7, $2 \mathrm{mM} \mathrm{MgCl}_{2}, 0.5 \mathrm{mM}$ EGTA, $1 \mathrm{mM}$ GTP and $20 \mathrm{mM}$ Taxol) at $35^{\circ} \mathrm{C}$ for $20 \mathrm{~min}$ to induce microtubule formation. To prepare CTTNBP2 proteins, COS1 cells were transfected with either WT or D570Y mutant expression plasmid. One day later, soluble total protein lysates containing CTTNBP2 proteins were collected from the supernatants after centrifugation at $16,000 \times \mathrm{g}$ for $20 \mathrm{~min}$ at $4{ }^{\circ} \mathrm{C}$ (table-top microcentrifuge, Heraeus Biofuge Fresco). Different amounts of protein lysates were incubated with or without microtubule at room temperature for $30 \mathrm{~min}$. The samples were then placed onto $100 \mu \mathrm{l}$ cushion buffer (60\% glycerol in GTB) and centrifuged at $100,000 \times \mathrm{g}$ for $40 \mathrm{~min}$ at room temperature. The pellet fraction is the microtubule binding fraction and the supernatant is the unbound fraction. The sample was then boiled in $2 \times$ sample buffer for $10 \mathrm{~min}$ and analyzed by immunoblotting.

\section{Mouse behavioral assays Open field}

The open field test was conducted as described previously $[6,12]$ to monitor locomotor activity and anxiety. Briefly, the test animal was placed in the center of a transparent acrylic box $(40 \times 40 \times 30 \mathrm{~cm})$ and allowed to freely explore the environment. The experiment was videotaped for $10 \mathrm{~min}$ from above the box. The central zone of the box was defined by a square $(20 \times 20 \mathrm{~cm})$ equidistant from the walls. The area of the defined central zone is equal to the sum of the four corners. To track and analyze the movement of the mice, the Smart Video Tracking System (Panlab) was employed. Total moving distance (to indicate locomotor activity) and the ratio of time spent at the center to that at the corner (to represent the degree of anxiety) were measured.

\section{Elevated plus maze}

A plus maze composed of two open arms and two closed arms $(30 \times 5 \mathrm{~cm})$ extending from a small central platform $(5 \times 5 \mathrm{~cm})$ was elevated from the floor to a height of 45.5 $\mathrm{cm}$ for the test. The test mouse was placed at the center of the platform and allowed to freely explore the environment for 10 min. The Smart Video Tracking System (Panlab) was used to track the movement of the animal. The percentages of time spent in open arms and closed arms were assessed.

\section{Reciprocal social interaction (RSI)}

Test mice were individually housed for approximately one week before the experiment. A stranger adult male mouse of the same age as test mice or one week younger was placed into the home cage of the test mouse for $10 \mathrm{~min}$. During the entire session, the lid of the cage was kept open to limit aggressive behaviors. Mouse behaviors were recorded by videotaping from above. The total time the test mouse spent sniffing the stranger mouse (headto-head, head-to-body and head-to-anogenital) was manually recorded to represent social interaction.

\section{Three-chambered test}

The three-chamber was performed as described previously $[10,12]$. In brief, the apparatus was a rectangular transparent plastic box $(17.5 \times 41.4 \times 22 \mathrm{~cm})$, with two dividing walls that separate the box into three equal chambers. Each dividing wall had a sliding entrance to connect different chambers. Two cylindrical wire cages $(10.5 \mathrm{~cm}$ in diameter and $11 \mathrm{~cm}$ in height) were put in the left and right chambers. The experiment comprised three 10-min sessions (habituation, sociability and novelty preference), with 5 min intervals between each session. During intervals, test mice were placed back in their home cage. In every session, the test mouse was placed into the central chamber and the two sliding doors were then simultaneously opened to allow the mouse to freely explore the whole environment. In the habituation session, both cylindrical wire cages were empty. In the sociability test session, an object $(\mathrm{Ob})$ was placed in one of the wire cages and a stranger mouse (S1) was placed in the other wire cage. In the social novelty preference session, the object was replaced by another stranger mouse (S2). Mouse behaviors were recorded by videotaping from above. Sniffing toward the cylindrical wire cages was considered as social interaction, which was quantified manually without knowing the genotype of mice. The value of $\left(\mathrm{T}_{\mathrm{S} 1}-\mathrm{T}_{\mathrm{Ob}}\right) /\left(\mathrm{T}_{\mathrm{S} 1}+\mathrm{T}_{\mathrm{Ob}}\right)$ was defined as the preference index of sociability. The value of $\left(\mathrm{T}_{\mathrm{S} 2}-\mathrm{T}_{\mathrm{S} 1}\right) /\left(\mathrm{T}_{\mathrm{S} 2}+\mathrm{T}_{\mathrm{S} 1}\right)$ was defined as novelty preference. $\mathrm{T}_{\mathrm{Ob}}$ is the interaction time with the object, $\mathrm{T}_{\mathrm{S} 1}$ is the interaction time with $\mathrm{S} 1$, and $\mathrm{T}_{\mathrm{S} 2}$ is the interaction time with $\mathrm{S} 2$.

\section{Statistical analyses}

Statistical analysis and graphical outputs were performed using PRISM 5.03 or 8.3 (Graphpad software). All sample sizes, statistical data and corresponding statistical methods are summarized in Tables S1 and S2. In brief, to compare multiple groups with one variant, one-way ANOVA with Bonferroni multiple comparison post hoc test was performed for normally distributed data and 
Kruskal-Wallis test with Dunn's multiple comparison test was performed for nonparametric distributed data. To compare multiple groups with two variants, twoway ANOVA was employed. To compare two groups of unrelated datasets, two-tailed Mann-Whitney test or unpaired $\mathrm{t}$ test was employed. In this report, $P<0.05$ is regarded as significant. For cumulative distribution analysis, a Kolmogorov-Smirnov (K-S) test was performed (https://www.aatbio.com/tools/kolmogorov-smirnov-ks-test-calculator). Outliers in the dataset were excluded using the "Identify outlier" tool in PRISM. All image analyses, including immunoblotting and morphometric analyses, were conducted using Image $(\mathrm{NIH})$. All morphometric and behavioral assay data were analyzed blind to minimize personal bias by relabeling the samples before being analyzed by another member of the laboratory. All statistical methods and results are summarized in Additional file 2: Table S1, Additional file 3: Table S2.

\section{Results \\ ASD-linked mutations alter dendritic spine formation and the subcellular distribution of CTTNBP2}

The ASD-linked mutations of CTTNBP2 we targeted are distributed along the entire short form of the protein (Fig. 1a, upper). Since human, mouse and rat CTTNBP2 proteins are highly conserved and the residues of ASDlinked mutations are identical among these three species (Additional file 1: Fig. S1), we used both rat and mouse models to investigate seven ASD-linked mutations of CTTNBP2. Monoallelic mutation of CTTNBP2 has been identified in patients. Thus, we overexpressed Cttnbp2 ASD-linked mutants in cultured neurons and used heterozygous knockin mutant mice to mimic the genetic condition of patients. The corresponding residues in mouse CTTNBP2 of the human mutations are R42W, A112T, M120I, G342R, P353A, R533* and D570Y (Fig. 1a, upper). Among these mutations, R533* mutation generated a truncated variant that lacks the C-terminal proline-rich domain. We expected R533* mutation would disrupt the interaction between CTTNBP2 and cortactin, impairing dendritic spine formation. For the remaining six mutations, there was no obvious clue to predict the impact of these mutations.

To characterize CTTNBP2, first we analyzed CTTNBP2 structure using IUPred2 (https://iupred2a. elte.hu/). Our results indicate that CTTNBP2 is an intrinsically unstructured molecule, apart from the $\mathrm{N}$-terminal coiled-coil region (Fig. 1a, lower). Next, we expressed the seven ASD-linked mutations of mouse Cttnbp2 in COS1 cells to confirm their expression (Fig. 1b). Since CTTNBP2 is critical for dendritic spine formation and maintenance $[5,20]$, we then investigated if these mutations alter dendritic spine formation.
To do that, we individually transfected the seven ASDlinked mutant proteins into wild-type (WT) cultured hippocampal neurons. Overexpressed WT CTTNBP2 formed puncta at dendritic spines and presented a filamentous pattern along axons of mature cultured neurons at 18 days in vitro (DIV) (Fig. 1c). Similar to WT CTTNBP2, the R42W, A112T, G342R and P353A mutant proteins were enriched at dendritic spines (Fig. 1c). However, the M120I and R533* mutant proteins were evenly distributed throughout neurons (Fig. 1c). Moreover, the D570Y mutant protein formed filamentous bundles along dendritic shafts and particularly along the proximal dendrites, a phenotype that differed from WT as well as those of the other mutants (Fig. 1c).

Next, we quantified dendritic spine density to evaluate the impact of ASD-linked mutations on excitatory synapse formation (Fig. 1c). Coexpression of GFP-actin was performed to outline neuronal morphology, including dendritic spines. We found that overexpressing WT CTTNBP2 slightly increased dendritic spine density relative to vector control. Compared to WT, expression of the R42W, M120I, G342R and R533* mutants reduced dendritic spine density in cultured neurons. In particular, the dendritic spine densities of the M120I and R533* mutant neurons were even lower than for neurons transfected with vector control (Fig. 1c). Thus, R42W and G342R might represent loss-of-function mutations that do not exert the same beneficial effect on spinogenesis as WT protein, whereas M120I and R533* may have dominant-negative effects that disrupt the function of endogenous CTTNBP2 (Fig. 1c, Table S1. Note that all statistical results in this report are summarized in Additional file 2: Table S1, Additional file 3: Table S2).

To further corroborate the effect of M120I and R533* on dendritic spine density, we performed patch clamp recording to measure the miniature excitatory postsynaptic current (mEPSC) of M120I or R533* mutant protein-expressing neurons. Compared with WT, expression of M120I or R533* mutant proteins reduced the frequency, but not amplitude, of mEPSCs (Fig. 1d), echoing the effect of M120I or R533* mutant proteins on dendritic spine formation. Though expression of the D570Y mutant protein did not alter dendritic spine density, we were intrigued by its unique filamentous pattern along dendritic shafts. Hereafter, we focus on further dissecting how the M120I, R533* and D570Y mutations alter CTTNBP2 distribution and function.

\section{M120I and R533* mutant proteins display reduced interactions with cortactin}

Since CTTNBP2 acts as a synaptic scaffold protein, we applied biochemical approaches to analyze the protein-protein interactions of Cttnbp2 mutants. The 

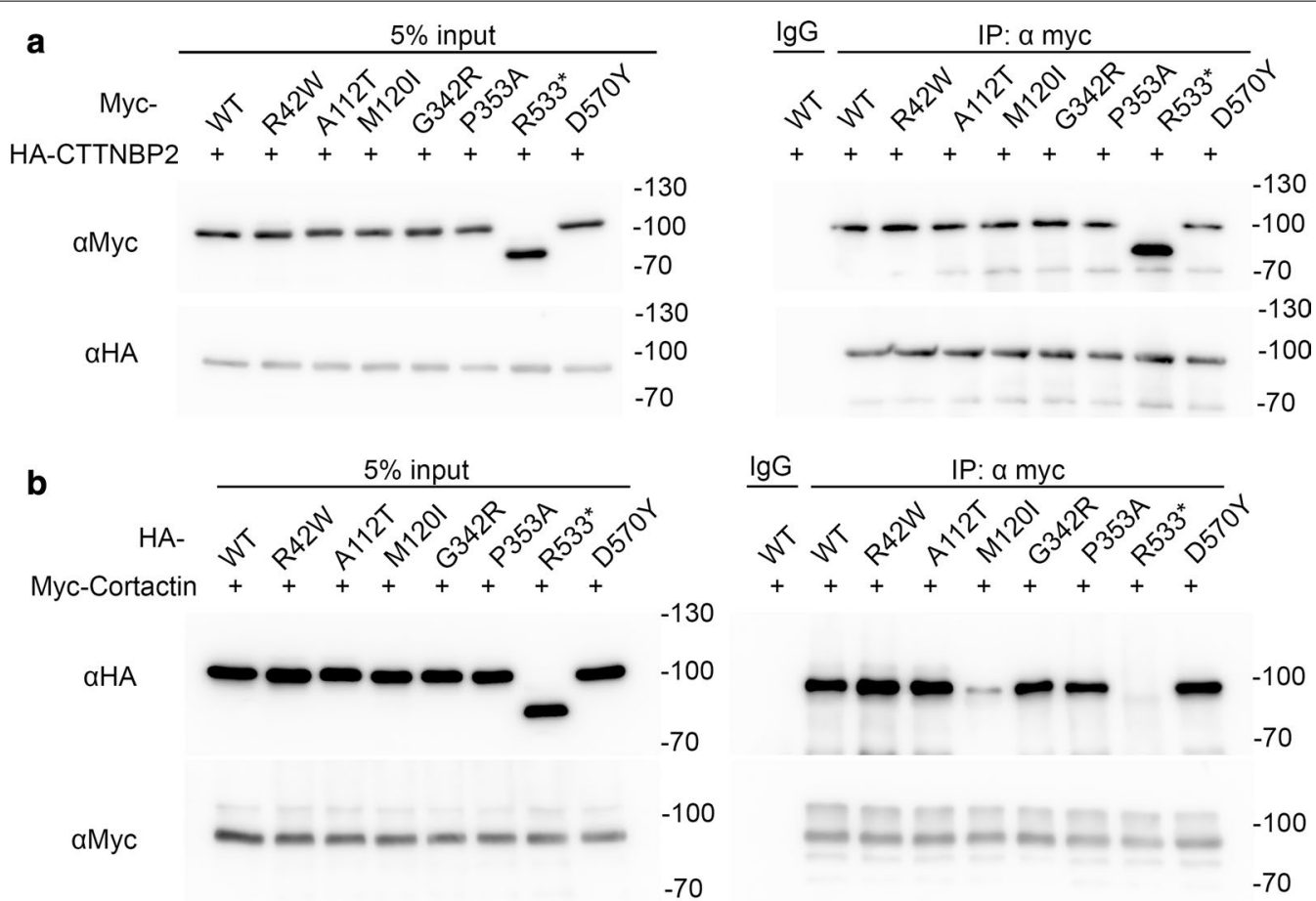

Fig. $2 \mathrm{M} 120$ and R533* mutations impair the C-terminal protein interactions of CTTNBP2. Co-immunoprecipitation (IP) using transfected COS1 cell lysates was performed to analyze the protein-protein interactions of seven ASD-linked mutations of CTTNBP2. a The interactions between HA-tagged WT CTTNBP2 and Myc-tagged ASD mutants. b The interaction between Myc-tagged cortactin and various HA-tagged CTTNBP2 constructs from transfected COS1 cells. Myc tag mouse antibody was used for IP, and both Myc and HA antibodies (from rabbit and rat, respectively) were used for immunoblotting, as indicated

M120I mutation is located in the N-terminal coiledcoil domain, which is involved in CTTNBP2 heteroand homo-oligomerization $[4,21]$. Both the R533* and D570Y mutations occur in the C-terminal P-rich region, which is required for cortactin interaction [5]. We used transfected COS1 cells to investigate if these mutations influence the interactions of CTTNBP2 with its binding partners, including cortactin and CTTNBP2 itself. We found that all Myc-tagged WT and the ASD-linked CTTNBP2 mutant proteins were able to co-immunoprecipitate with WT CTTNBP2 from COS1 lysates (Fig. 2a), suggesting that ASDlinked mutations do not alter CTTNBP2 oligomerization. To further evaluate the interaction between CTTNBP2 and cortactin, we cotransfected Myctagged cortactin and various HA-tagged CTTNBP2 constructs into COS1 cells. Myc-tag antibody was then used to immunoprecipitate cortactin. As anticipated, R533* mutant protein was not present in the precipitate, indicating that $\mathrm{R} 533^{*}$ mutation indeed disrupts the interaction between CTTNBP2 and cortactin. However, unexpectedly, we found that the M120I mutant protein exhibited greatly reduced interaction with cortactin (Fig. 2b), indicating that this N-terminal M120I mutation influences the C-terminal structure or its accessibility for cortactin interaction.

\section{Cortactin overexpression ameliorates dendritic spine deficits caused by M120I and R533* mutations}

Our previous report indicated that cortactin is a critical downstream factor of CTTNBP2 to control dendritic spine formation. Cortactin overexpression rescues dendritic spine defects caused by Cttnbp2 knockdown [5]. Since the M120I and R533* mutant proteins are both defective in their interaction with cortactin, we hypothesized that cortactin overexpression would rescue the dendritic spine defects caused by these two mutations. To test that possibility, we triply transfected GFP-actin, HA-tagged CTTNBP2 variants (including WT, M120I and R533*) and Myc-tagged cortactin or vector control into cultured hippocampal neurons (Fig. 3a). Indeed, cortactin overexpression increased the dendritic spine density of M120I- or R533*-expressing neurons (Fig. 3a), evidencing an important role for cortactin-mediated dendritic spine formation in the ASD pathogenic pathway induced by CTTNBP2 mutations. 


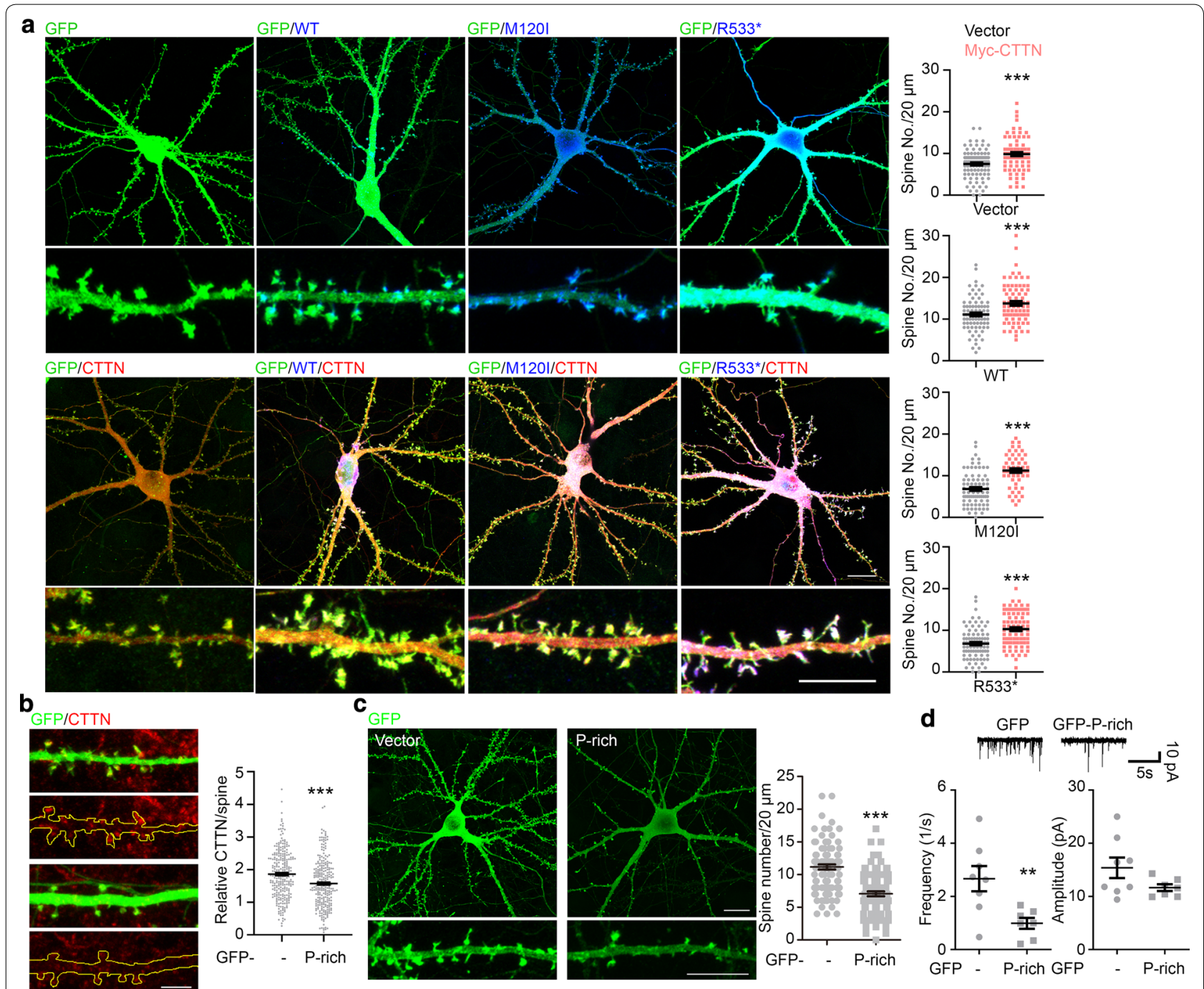

Fig. 3 Interaction with cortactin via the C-terminal P-rich domain of CTTNBP2 is essential for dendritic spine formation and activity. a Cortactin overexpression rescues dendritic spine density in neurons expressing M120l or R533* mutant protein. HA-tagged WT, M120l or R533* CTTNBP2 was cotransfected with Myc-tagged cortactin (CTTN) and GFP-actin. In M120l- and R533*-transfected neurons, cortactin expression rescues dendritic spine density. Quantification results of dendritic spine density are shown. $\mathbf{b}$ Overexpression of the GFP-P-rich construct reduced the synaptic distribution of cortactin in mature cultured hippocampal neurons. The signal of cortactin in individual spines was normalized with the signal for cortactin in the soma to represent synaptic enrichment of cortactin. c Overexpression of the GFP-P-rich construct reduces dendritic spine density. d GFP-P-rich overexpression reduces mEPSC frequency, but not amplitude. GFP was used as a control in (b)-(d). Data represent mean \pm SEM from three independent experiments. Each dot in $(\mathbf{a}, \mathbf{c}),(\mathbf{b})$ and $(\mathbf{d})$ represents an individual dendrite, spine or, neuron, respectively. Two-tailed unpaired $t$ tests were performed. All statistical data and exact sample sizes are available in Additional file 2: Table $S 1 .{ }^{*} P<0.05 ;{ }^{* *} P<0.01 ;{ }^{* *} P<0.001 ; \mathrm{ns}$, not significant. Scale bar: a upper, $20 \mu \mathrm{m}$; lower, $10 \mu \mathrm{m}$; b $5 \mu \mathrm{m}$; c upper, $20 \mu \mathrm{m}$; lower, $10 \mu \mathrm{m}$

\section{The P-rich domain of CTTNBP2 is critical for controlling} dendritic spine density and synaptic activity

To further validate the significance of the interaction between cortactin and CTTNBP2 for dendritic spine formation, we overexpressed the GFP-tagged P-rich (GFP-P-rich) domain of CTTNBP2 to disrupt the interaction between endogenous CTTNBP2 and cortactin in cultured neurons. We found that GFP-P-rich overexpression reduced the synaptic distribution of cortactin (Fig. 3b). Consistent with the essential role of cortactin in dendritic spine formation, GFPP-rich overexpression also resulted in decreased dendritic spine density (Fig. 3c). Moreover, measurement of mEPSCs revealed a reduction in frequency but not amplitude due to GFP-P-rich overexpression (Fig. 3d). These findings support that the interaction 

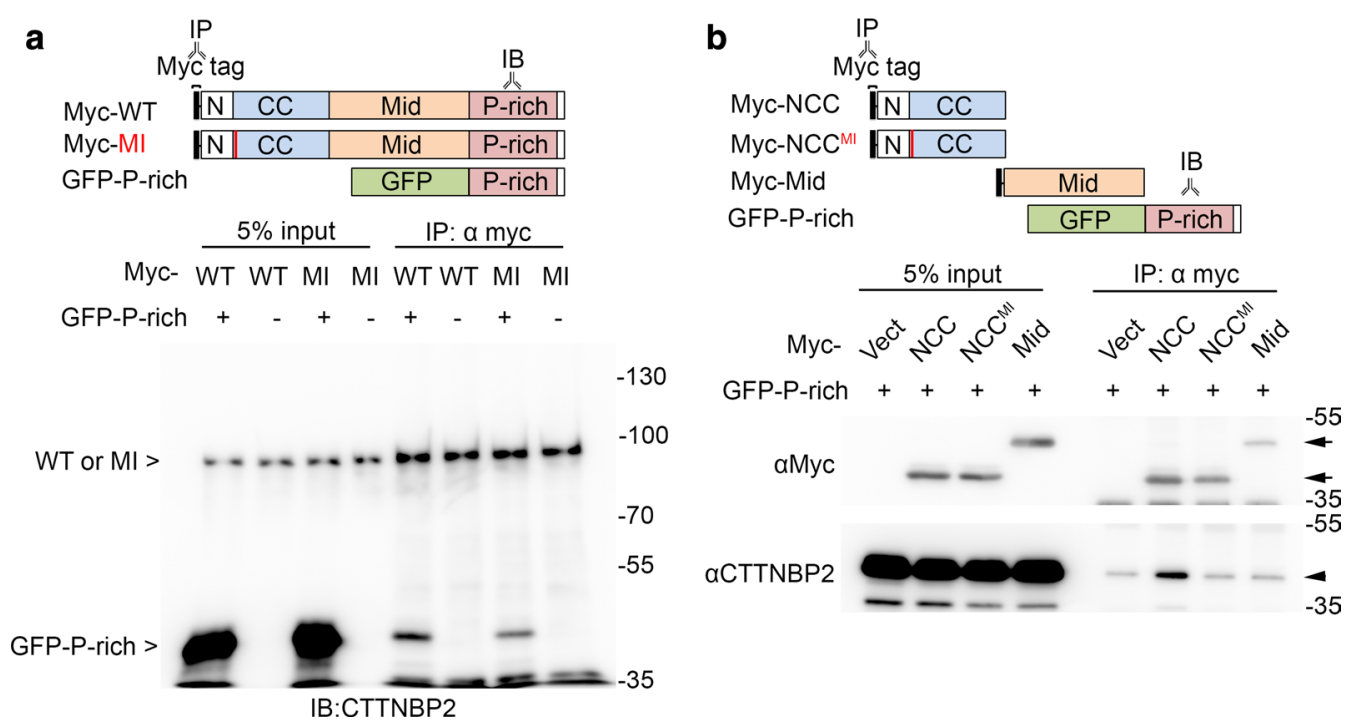

Fig. 4 M120l mutation impairs the N-C terminal interaction of CTTNBP2. a M120I mutation compromises the association of full-length CTTNBP2 with GFP-P-rich fragments. Top: schematics of constructs. Bottom: co-immunoprecipiation (IP) results. Full-length Myc-tagged wild type (Myc-WT) or M120I (Myc-MI) CTTNBP2 proteins were precipitated using Myc tag antibody. Antibody recognizing the C-terminal CTTNBP2 region was used in immunoblotting to recognize all Myc-WT, Myc-Ml and GFP-P-rich constructs. GFP vector was used as negative control. b M120l mutation compromised the association of GFP-P-rich fragments with the NCC fragment of CTTNBP2. Top: schematics of constructs. Bottom: Co-immunoprecipitation results. Myc-tagged NCC domains (either wild type Myc-NCC or M1201-mutant Myc-NCC ${ }^{\mathrm{MI}}$ ) were precipitated by Myc tag antibody. Vector only or Mid domain was used as a negative control. Myc and CTTNBP2 antibodies were used for immunoblotting. Arrows point to Myc-tagged constructs, whereas arrowhead indicates GFP-P-rich construct

between cortactin and the C-terminal P-rich domain of CTTNBP2 is fundamental to dendritic spine formation and synaptic activity.

\section{M120l mutation disrupts the $\mathrm{N}-\mathrm{C}$ terminal interaction of CTTNBP2}

The M120 residue is located at the beginning of the coiled-coil (CC) domain, a region far from the C-terminal cortactin binding region (Fig. 1a). We were surprised to find that the M120I mutation also diminished the CTTNBP2-cortactin interaction. Since CTTNBP2 is an intrinsically unstructured molecule (Fig. 1a), it implies that CTTNBP2 proteins are inherently flexible. Accordingly, we postulated that a $\mathrm{N}-\mathrm{C}$ terminal interaction modulates the $\mathrm{C}$-terminal protein-protein interactions of CTTNBP2. To validate the $\mathrm{N}-\mathrm{C}$ interaction, we cotransfected full-length Myc-tagged WT or M120I mutant protein with GFP-P-rich fragments into COS1 cells and conducted immunoprecipitation with Myc tag antibody (Fig. 4a). Indeed, GFP-P-rich fragments coimmunoprecipitated with WT CTTNBP2, whereas association of the M120I mutant with GFP-P-rich fragments was reduced relative to WT (Fig. 4a). This outcome indicates that the M120I mutation interfered with the $\mathrm{N}-\mathrm{C}$ interaction. To further confirm this point, we co-transfected Myc-tagged WT N-terminal coiled-coil domain
$\left(\mathrm{NCC}^{\mathrm{WT}}\right)$ or the NCC domain with the M120I mutation $\left(\mathrm{NCC}^{\mathrm{MI}}\right)$ together with GFP-P-rich fragments. We observed a much stronger interaction of $\mathrm{NCC}^{\mathrm{WT}}$ with GFP-P-rich fragments than NCC ${ }^{\mathrm{MI}}$ (Fig. 4b). The interaction was specific because the Myc-tagged Mid domain did not have the same ability to precipitate the GFP-Prich fragments (Fig. 4b). These results indicate that the $\mathrm{N}$-terminal coiled-coil domain of CTTNBP2 interacts with the protein's $\mathrm{C}$-terminal P-rich domain, and that this interaction is compromised by M120I mutation.

Based on these analyses, we suggest that the impaired dendritic spine formation caused by the M120I and R533* mutant proteins is due to their incapacity to interact with cortactin. However, different mechanisms are responsible for disrupting the interaction between these two mutant CTTNBP2 proteins and cortactin.

\section{D570Y mutation enhances the microtubule association of CTTNBP2 and microtubule stability}

We then investigated if and how the D570Y mutation influences CTTNBP2 function. Since CTTNBP2 also associates with microtubule and distributes along the dendritic shaft mainly before mature spine formation [21], the distribution of D570Y mutant proteins at the dendritic shaft of mature neurons (Fig. 1c) suggests a potential alteration of the interaction between 


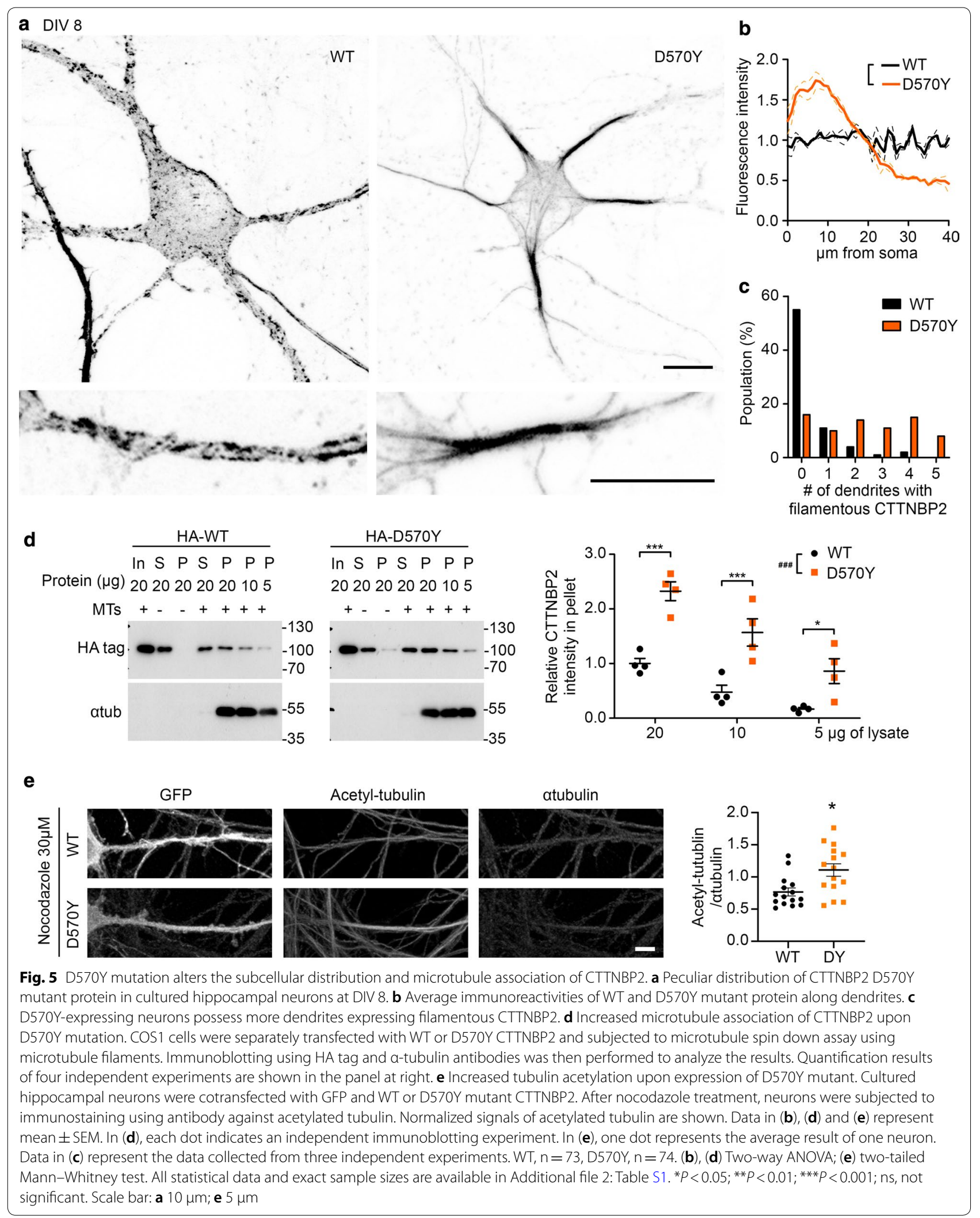


microtubule and CTTNBP2 protein. To investigate that possibility, we first investigated the distribution of D570Y mutant protein in immature hippocampal neurons. Similar to our previous finding [21], WT CTTNBP2 formed punctate aggregates along dendrites at DIV 8 (Fig. 5a, left). However, D570Y mutant protein preferentially formed filamentous structures and exhibited a peculiar accumulation in proximal dendrites (Fig. 5a). We quantified the intensity of CTTNBP2 signal along dendrites and found that, unlike WT protein, D570Y mutant protein strongly accumulated along dendrites within a distance of $20 \mu \mathrm{m}$ from the soma (Fig. 5b). Furthermore, the D570Y mutant-expressing neurons tended to have multiple dendrites with CTTNBP2 filaments (Fig. 5a, c). Thus, our results indicate that the D570Y mutation alters the subcellular distribution of CTTNBP2 in neurons.

To investigate if D570Y mutation results in a higher affinity to bind microtubule, we performed a microtubule binding protein spin-down assay. COS1 lysates that expressed WT or D570Y mutant CTTNBP2 proteins were mixed with preassembled microtubule filaments and subjected to centrifugation to examine if microtubule can bring down more D570Y mutant protein relative to WT. By applying three different lysate amounts (i.e. 5, 10 and $20 \mu \mathrm{g}$ of protein) in our microtubule spin-down assay, we found that both WT and D570Y mutant protein exhibited a dosage-dependent microtubule association, but amounts of microtubule binding by D570Y mutant protein were 2.5-4-fold higher than those displayed by WT protein (Fig. $5 \mathrm{~d}$ ).

Given the higher affinity for microtubule of D570Y mutant protein relative to $\mathrm{WT}$, it is likely to increase microtubule stability. To monitor microtubule stability in neurons, we transfected WT or D570Y mutant protein into cultured hippocampal neurons and added nocodazole to disrupt the microtubule network. Then, we analyzed the level of acetylated tubulin, a stable tubulin marker, within the proximal dendrites of our cultured hippocampal neurons. Compared with neurons transfected with WT CTTNBP2, neurons transfected with D570Y mutant protein displayed higher levels of acetylated tubulin within proximal dendrites (Fig. 5e), supporting that the D570Y mutation increases microtubule stability.

Thus, our experiments have shown that D570Y mutant protein has a higher affinity for microtubule, resulting in increased microtubule stability.

\section{D570Y mutation reduces the dendritic spine distribution of CTTNBP2 and dendritic spine density}

Since D570Y mutant protein displayed greater ability to bind microtubule and given that microtubule is mainly present in the dendritic shaft but not dendritic spines, we expected that the D570Y mutation would result in CTTNBP2 being retained in the dendritic shaft, thereby reducing the synaptic distribution of CTTNBP2 in mature neurons. However, our data shown in Fig. 1c suggest that the D570Y mutation does not overly perturb dendritic spine formation. Potentially, self-oligomerization with endogenous WT CTTNBP2 enables exogenous D570Y mutant protein to enter dendritic spines and maintain dendritic spine formation. If this speculation is correct, a reduction of endogenous WT CTTNBP2 should result in impaired dendritic spine distribution of D570Y mutant protein. To investigate that possibility, we applied the previously-established knockdown construct BP2-miR to reduce endogenous WT CTTNBP2 protein [5]. BP2-miR-resistant WT and D570Y mutant proteins were then cotransfected with BP2-miR or control vector Ctrl-miR into neurons (Fig. 6a). We found that compared to WT CTTNBP2, expression of the D570Y mutant protein indeed resulted in a reduced dendritic spine density in BP2-miR-expressing neurons (Fig. 6b). This finding is consistent with our speculation that endogenous WT CTTNBP2 neutralizes the impact of the D570Y mutation.

Next, we applied line-scanning from the tip to the base of dendritic spines to quantify the intensity of WT and D570Y mutant proteins in dendritic spines (Fig. 6c, d). Immunoreactivity of WT CTTNBP2 was enriched at the tips of dendritic spines (within 0-0.5 $\mu \mathrm{m}$ from the tip) and declined as distance from the tip increased, particularly within the region of 1-1.5 $\mu \mathrm{m}$ from the tip in both BP2-miR- and Ctrl-miR-expressing neurons (Fig. 6c, d). In contrast, we noted a high level of D570Y mutant protein expression within the 1-1.5 $\mu \mathrm{m}$ region (Fig. 6c, d). In fact, signal of D570Y mutant protein was much lower around the tips of dendritic spines from BP2-miRexpressing neurons (Fig. 6d). In Ctrl-miR-expressing neurons, synaptic distributions of WT and D570Y mutant protein were comparable (Fig. 6c). Together, these results indicate that the D570Y mutation indeed reduces synaptic targeting of CTTNBP2.

\section{D570Y and M120I mutations result in dendritic spine deficits in mice}

To further investigate the physiological relevance of ASDlinked mutations of Cttnbp2, we employed CRISPR/Cas9 genomic editing technology to generate M120I, R533* and D570Y knockin mice (Fig. 7a-d). Results for R533* mice have been reported previously [20]. Since only one of the two CTTNBP2 alleles in ASD patients carries one of these three mutations, we used heterozygous KI mice to mimic patient conditions. Hereafter, M120I and D570Y mutant mice denote these heterozygous 


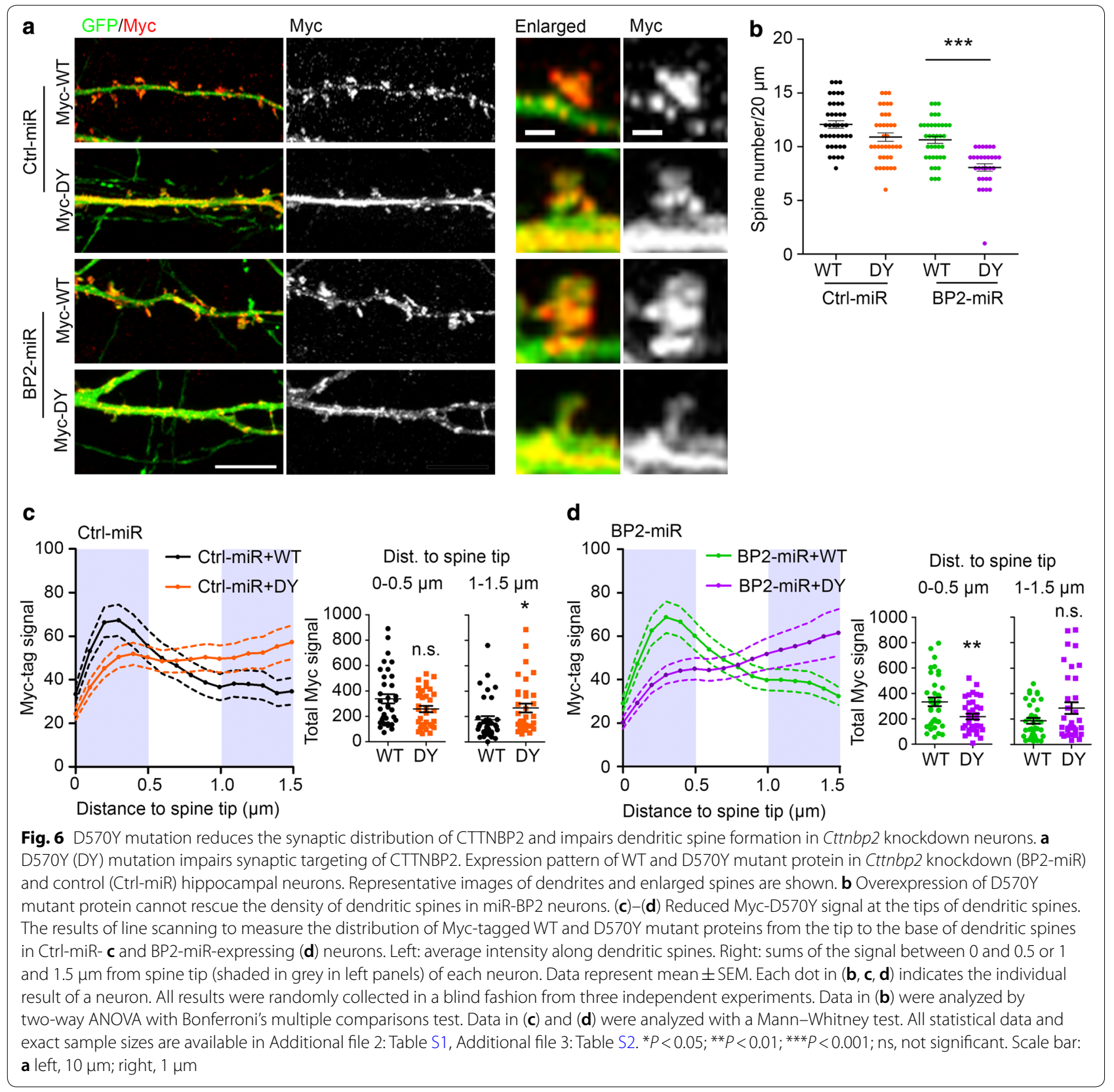

knockin mice. Note, to avoid potential off-target effects, all mutant mice were backcrossed to WT mice for more than six generations before undergoing experimentation.

Similar to Cttnbp2 deletion and R533* mutant mice [20], neither M120I nor D570Y mutant mice displayed obvious anatomical defects in their brains (Fig. 7b, d). To investigate the dendritic spine features of these mutant mice, we crossed the mice with Thy1-YFP-H transgenic mice to outline neuronal morphology of forebrain projection neurons. Similar to our in vitro results, M120I mutant mice exhibited reduced density, width and length of dendritic spines in dorsal hippocampal CA1 neurons (Fig. 7e, f). We also compared the ratio of the width to the length $(\mathrm{W} / \mathrm{L})$ of individual spines and did not observe a statistical difference between M120I and WT neurons (Fig. 7g), suggesting a proportional shrinkage of dendritic spines in M120I mutant neurons. For D570Y mutant mice, although spine width and length were not altered (Fig. $7 \mathrm{~h}-\mathrm{j}$ ), dendritic spine density was reduced compared to that of WT littermates (Fig. $7 \mathrm{~h}-\mathrm{i}$ ). Thus, these results confirm that, similar to Cttnbp2 deletion and R533* 


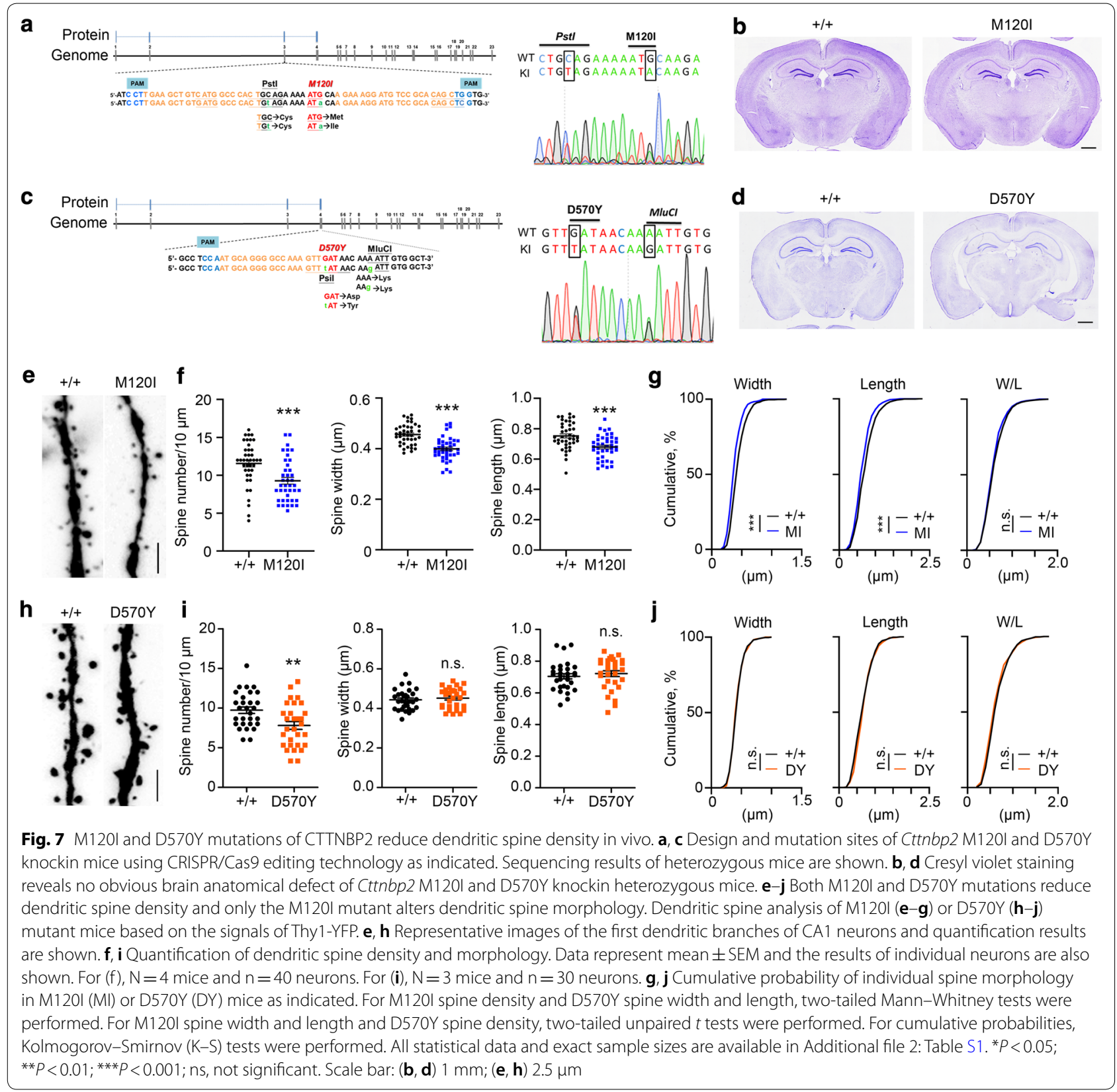

mutation, the M120I and D570Y mutations impair dendritic spine formation in vivo.

\section{M120l mutant mice exhibit impaired social interactions and neuronal activation}

Recently, we reported that Cttnbp2-/-, $+/-$ and R533* mutant mice all exhibit impaired social interaction behaviors [20], supporting a role for CTTNBP2 in regulating social behaviors. Here, we further assessed M120I mutant mice as a model to investigate if reduced social interaction is a typical trait induced by ASD-linked mutations in the CTTNBP2 gene. We employed open field, elevated plus maze, reciprocal social interaction (RSI) and three-chamber tests to analyze M120I mutant mice (Fig. 8a). Similar to our report on R533* mutant mice [20], M120I mutant mice did not exhibit defects in their responses to open field and elevated plus maze tests (Fig. 8b, c), suggesting that M120I mutation does not influence locomotor activity or anxiety. For RSI, M120I mutant mice spent less time approaching the stranger mouse compared to wildtype littermates (Fig. 8d). In the sociability session of 


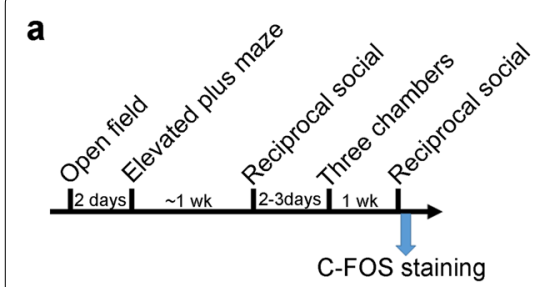

e Sociablilty
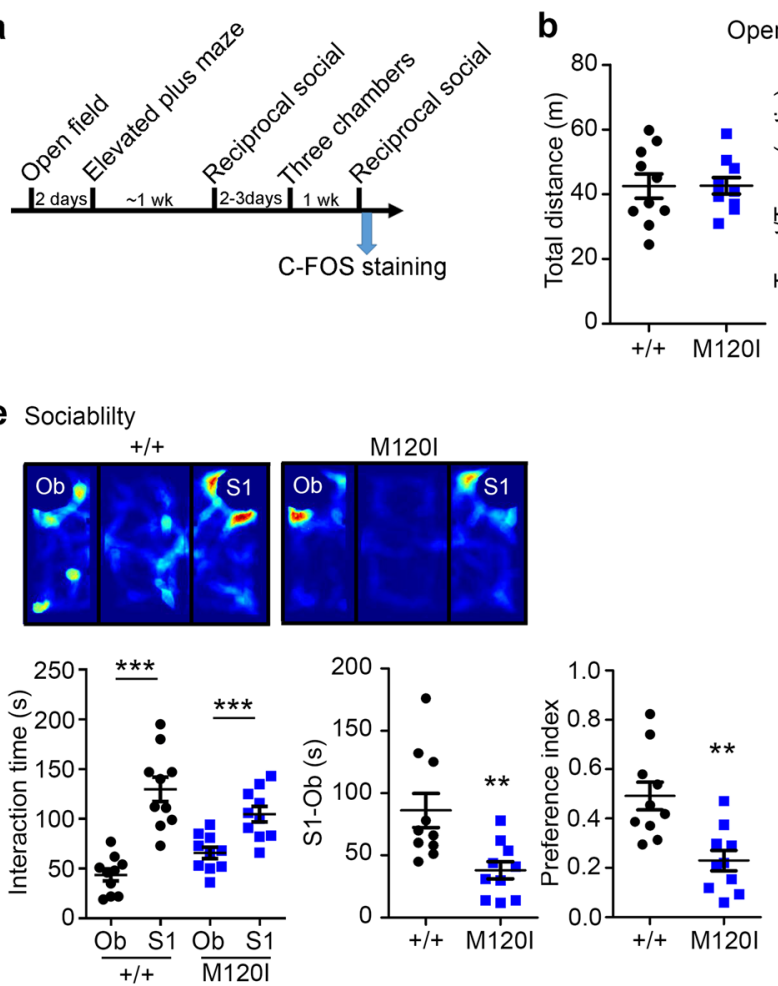
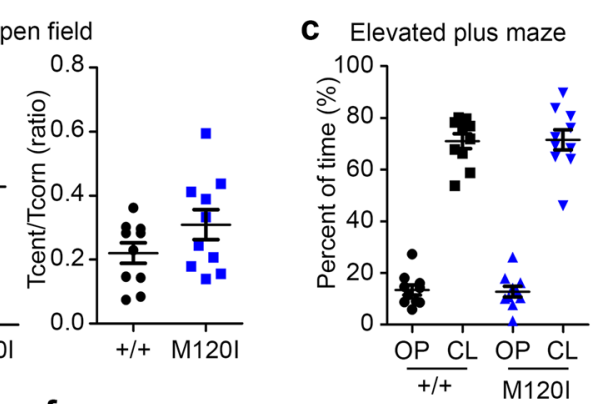

d

RSI

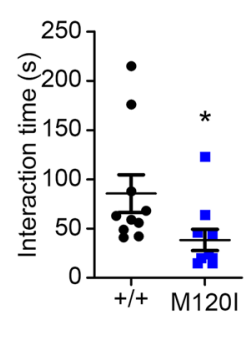

f Novelty preference
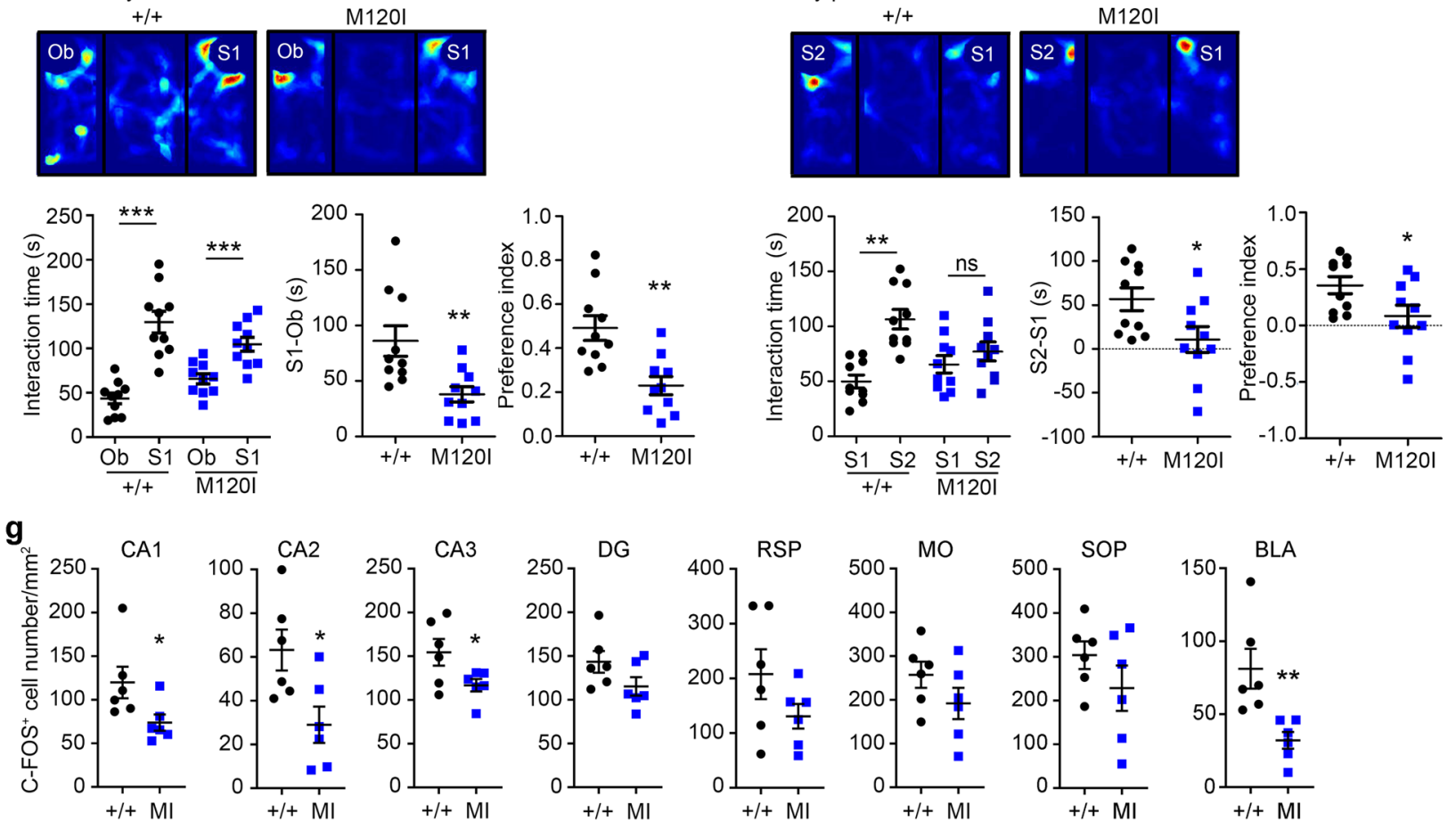

Fig. 8 Cttnbp2 M120l mutant mice exhibit reduced social interaction and neuronal activation. a Flowchart of behavioral assays used in this report. Two hours after the last assay, mouse brains were fixed and subjected to C-FOS staining. b Open field. c Elevated plus maze. $\mathbf{d}$ Reduced reciprocal social interaction (RSI) in M120l mutant mice. e Reduced sociability of $\mathrm{M} 120 \mathrm{I}$ mutant mice in three-chamber test. f Reduced novelty preference of M120l mutant mice in three-chamber test. Heat maps of movement paths of mice, actual interaction time, the difference of interaction time between the left and right chambers, and preference index are shown. g C-FOS staining of M120l mutant mice after social stimulation. DG dentate gyrus, RSP retrosplenial cortex, MO motor cortex, SOP somatosensory cortex, BLA basal lateral amygdala. Data represent mean \pm SEM and the results of individual mice are shown. d Two-tailed Mann-Whitney test. e-f A paired $t$ test was used to compare total interaction times for the object (Ob) and stranger 1 (S1) or for S1 and stranger 2 (S2). To compare interaction times or preference indexes, an unpaired t-test or Mann-Whitney test was used. For (g), an unpaired $t$-test or Mann-Whitney test was used. All statistical data and exact sample sizes are available in Additional file 2: Table S1. ${ }^{*}, p<0.05 ;{ }^{*}, p<0.01 ;{ }^{* *}, p<0.001 ;$ ns, not significant

three-chamber test, although M120I mutant mice spent more time interacting with the stranger mouse (S1) rather than the object $(\mathrm{Ob})$, both the time difference (i.e. S1-O) and the corresponding preference index of M120I mutant mice were lower than that of WT littermates (Fig. 8e). In the novelty preference session, wild-type littermates spent more time interacting with stranger mouse 2 (S2), but M120I mutant mice did not exhibit a preference for either S1 or S2 (Fig. 8f). These behavioral assays support that M120I mutation indeed results in reduced social behaviors and impaired social novelty preference, representing a core symptom of ASD.

Finally, we investigated if the behavioral deficits of M120I mutant mice are relevant to impaired neuronal activation. Two hours after our RSI test (Fig. 8a), we performed C-FOS staining on the test mice to monitor neuronal activation upon social stimulation. Similar to 
Cttnbp $2^{-/-}$mice [20], we found that neuronal activity of M120I mutant mice was lower at the CA1, CA2 and CA3 regions of hippocampus and basolateral amygdala compared to WT littermates (Fig. 8g).

In conclusion, together with our recently published results on R533* mutant mice [20], the findings presented in the current study suggest that ASD-associated Cttnbp 2 mutations indeed reduce dendritic spine density and neuronal activation and impair social behaviors in mice.

\section{Discussion}

In this report, we collectively studied seven ASD-linked mutations of Cttnbp 2 from the aspects of biochemistry, cell biology and physiology. Based on our analyses, we characterized the effect of three potential diseasecausative mutations, i.e. M120I, R533* and D570Y. These three mutations resulted in prominent dendritic spine defects in cultured hippocampal neurons, as well as in mouse brains. However, the mechanisms underlying the effects of these three mutations on dendritic spine formation differ, representing an illustrative example of how divergent mechanisms contribute to ASD etiology, even for mutations of the same gene, yet result in convergent outcomes.

Both the M120I and R533* mutations impair interactions of CTTNBP2 with cortactin, but the underlying mechanisms differ. R533* mutant protein lacks the C-terminal P-rich domain of CTTNBP2, so it lacks the ability to interact with cortactin. We found that overexpression of cortactin rescues the dendritic spine defects caused by R533* mutation, confirming a critical role for cortactin in the CTTNBP2 pathway. Since CTTNBP2 acts as a synaptic scaffold to regulate synaptic targeting of other proteins, such as SHANKs, NMDAR and protein phosphatase 2A regulatory subunit STRN [20], it is likely that the downstream effectors of CTTNBP2 and cortactin also crosstalk to other CTTNBP2-regulated proteins and consequently control dendritic spine formation.

The M120I mutation disrupts the $\mathrm{N}-\mathrm{C}$ terminal interaction of CTTNBP2 proteins, impeding cortactin interaction. Cortactin overexpression also rescued the dendritic spine deficits caused by M120I mutation, further confirming the involvement of cortactin in how CTTNBP2 controls dendritic spine formation and neuronal function. Certainly, it is possible that the disrupted $\mathrm{N}-\mathrm{C}$ interaction also influences other protein-protein interactions of CTTNBP2 and impairs coordination of cortactin and other CTTNBP2-interacting proteins. Apart from its $\mathrm{N}$-terminal CC domain, CTTNBP2 is intrinsically unstructured, providing the flexibility necessary for intra- and/or inter-molecular N-C interactions.
However, this flexibility also makes it difficult to conduct X-ray crystallography on CTTNBP2. We have repeatedly tried to purify full-length recombinant CTTNBP2 protein. Perhaps due to its unstructured nature, the recombinant protein is unstable in bacterial expression systems. Consequently, as yet, it is completely unknown how the $\mathrm{N}$ - and C-terminal regions of CTTNBP2 interact with each other and how the $\mathrm{N}$-terminal region of CTTNBP2 can regulate the interaction between the $\mathrm{C}$-terminal P-rich domain and cortactin.

Here, we have reported that a $\mathrm{N}-\mathrm{C}$ terminal interaction facilitates the interaction of CTTNBP2 C-terminal with cortactin, with this latter being disrupted by M120I mutation. This kind of facilitation is not rare in cytoskeleton regulators. For instance, N-WASP is a core signal transducer that receives signal from CDC42 and directs Arp2/3-dependent actin polymerization [1]. The intramolecular $\mathrm{N}-\mathrm{C}$ terminal interaction of N-WASP occludes the Arp2/3 binding site to inhibit actin polymerization [16]. Similarly, the microtubule binding and stabilizing property of $\mathrm{mPar} 3$ is facilitated by an intramolecular $\mathrm{N}-\mathrm{C}$ terminal interaction [3]. Intermolecular oligomerization of mPar competes for the intramolecular $\mathrm{N}-\mathrm{C}$ terminal interaction, exposing the microtubulebinding domain of mPar3 [3]. Our study on CTTNBP2 provides a new example of a cytoskeleton regulator that is modulated by its N-C interaction.

Although the D570Y mutation also reduced dendritic spine density, the underlying mechanism is different from those of the M120I and R533* mutations. Its higher affinity for microtubule results in this variant preferentially staying in the dendritic shaft rather than moving into dendritic spines. The reduction of CTTNBP2 protein levels at dendritic spines resembles the outcomes of Cttnbp 2 knockdown or knockout, resulting in lower dendritic spine density. However, apart from dendritic spine deficits, the binding of D570Y mutant protein to microtubule along the dendritic shaft and consequently increased microtubule stability may contribute to other phenotypes arising from D570Y mutation. This latter possibility requires further investigation. Interestingly, residue D570 is located very close to the $\mathrm{C}$-terminal end of the short form of CTTNBP2 (630 amino acid residues) [5]. Our previous data showed that the middle unstructured region of CTTNBP2 is required for association with microtubule [21]. Thus, the C-terminal region of CTTNBP2 may also regulate the protein interactions mediated by its middle region, which likely relates to the intrinsically unstructured nature of CTTNBP2. Our study implies that the protein structure of CTTNBP2 is very flexible and exhibits complex self-interactions and regulation. 
In this report, we have primarily focused on dendritic spine defects, with the R533* and M120I mutations resulting in the most severe phenotypes. For other investigated mutations, such as R42W and G342R, their impact on dendritic spine density was much milder. Unlike for WT protein that slightly increased dendritic spine density, overexpression of R42W and G342R mutants was unable to do so. This outcome indicates that R42W and G342R likely represent loss-of-function mutations. Apart from its influence on dendritic spines, CTTNBP2 also regulates dendritic arborization [21]. Since CTNNBP2 is also present along the axonal shaft, it would also be meaningful to investigate if these ASDlinked mutations influence dendritic arborization and/ or axonal growth, which may also contribute to ASD phenotypes.

Taken together with our previous study [20], we have now analyzed the behavioral features of Cttnbp $2^{-/-}, C t t n b p 2^{+/-}$, R533* and M120I mutant mice. Reduced social interactions with strangers in reciprocal social interaction and three-chamber tests are common defects shared among all of these examined mutant mouse lines. Since defective social interaction is one of the core symptoms of ASD, our mouse studies support that CTTNBP2 mutation is at least partially responsible for ASD. Despite these common social defects, other behavioral deficits are not exhibited by all of the examined mouse lines. For instance, $C t t n b p 2^{-/-}$mice exhibit the most severe behavioral abnormalities, including hyperactive locomotion, anxiolytic behaviors in elevated plus maze and marble burying tests, and impaired contextual memory in novel object recognition [20]. However, Cttnbp 2 ${ }^{+/-}$, R533* and M120I mutant mice did not display any noticeable phenotype in terms of locomotion or anxiety. Thus, it would be informative to correlate patients' mutations and symptoms with the phenotypes displayed by our mouse models. Such analysis would provide useful information as to whether mouse behavioral assays, at least for Cttnbp 2 mutant mice, are relevant to patients' conditions and could serve as appropriate models for etiological and therapeutic studies.

\section{Supplementary information}

Supplementary information accompanies this paper at https://doi. org/10.1186/s40478-020-01053-x.

Additional file 1: Fig. S1 Amino acid sequence alignment of human, rat and mouse CTTNBP2.

Additional file 2: Table S1 contains all statistical methods and results, except Fig. 5B.

Additional file 3: Table S2 contains the statistical results of Fig. 5B.

\section{Acknowledgement}

We thank the Transgenic Core Facility (supported by Academia Sinica, AS-CFII-108-104), Ms. Huei-Fang Wu at the Neuroscience Core Facility Core (supported by Academia Sinica, AS-CFII-108-106) and the Animal Facility of the Institute of Molecular Biology, Academia Sinica, for excellent technical assistance. Dr. John O'Brien conducted English editing and members of Dr. Yi-Ping Hsueh's laboratory relabeled samples for blind experiments.

\section{Authors' contributions}

P-YS conceptualization, methodology, investigation and writing; B-YH investigation and writing; C-YT investigation and writing; $C-A L$ investigation and writing; BEC investigation and writing; Y-PH conceptualization, writing, funding acquisition, supervision and project administration. All authors read and approved the final manuscript.

\section{Funding}

This work was supported by grants from Academia Sinica (AS-IA-106-L04) and the Ministry of Science and Technology (MOST 108-2311-B-001-008-MY3 and 108-2321-B-001-002) to Y.-P. Hsueh.

\section{Availability of data and materials}

Data generated or analyzed during this study are included in this published article and its supplementary information files.

\section{Consent for publication}

Not applicable.

\section{Ethics approval and consent to participate}

Human related study was not performed in this report. For animal study, all experiments were performed with the approval of the Academia Sinica Institutional Animal Care and Utilization Committee (protocol No 11-12-294 and 12-10-414).

\section{Competing interests}

The authors declare no conflict of interests.

\section{Author details}

${ }^{1}$ Molecular and Cell Biology, Taiwan International Graduate Program, Institute of Molecular Biology, Academia Sinica and Graduate Institute of Life Sciences, National Defense Medical Center, Taipei, Taiwan, ROC. ${ }^{2}$ Institute of Molecular Biology, Academia Sinica, Taipei, Taiwan, ROC. ${ }^{3}$ Centre for Research in Neuroscience, McGill University Health Centre, Montreal General Hospital, Montréal, QC H3G 1A4, Canada. ${ }^{4}$ Departments of Medicine and Neurology \& Neurosurgery, McGill University, Montréal, QC, Canada.

Received: 24 June 2020 Accepted: 10 October 2020

Published online: 09 November 2020

References

1. Alekhina O, Burstein E, Billadeau DD (2017) Cellular functions of WASP family proteins at a glance. J Cell Sci 130:2235. https://doi.org/10.1242/ jcs.199570

2. Bai D, Yip BHK, Windham GC, Sourander A, Francis R, Yoffe R, Glasson E, Mahjani B, Suominen A, Leonard $\mathrm{H}$ et al (2019) Association of genetic and environmental factors with autism in a 5-country cohort. JAMA Psychiatry 76:1035-1043. https://doi.org/10.1001/jamapsychiatry.2019.1411

3. Chen S, Chen J, Shi H, Wei M, Castaneda-Castellanos DR, Bultje RS, Pei X, Kriegstein AR, Zhang M, Shi SH (2013) Regulation of microtubule stability and organization by mammalian Par3 in specifying neuronal polarity. Dev Cell 24:26-40. https://doi.org/10.1016/j.devcel.2012.11.014

4. Chen YK, Chen CY, Hu HT, Hsueh YP (2012) CTTNBP2, but not CTTN$B P 2 N L$, regulates dendritic spinogenesis and synaptic distribution of the striatin-PP2A complex. Mol Biol Cell 23:4383-4392

5. Chen YK, Hsueh YP (2012) Cortactin-binding protein 2 modulates the mobility of cortactin and regulates dendritic spine formation and maintenance. J Neurosci 32:1043-1055. https://doi.org/10.1523/JNEUR OSCI.4405-11.2012 
6. Chung WC, Huang TN, Hsueh YP (2011) Targeted deletion of CASKinteracting nucleosome assembly protein causes higher locomotor and exploratory activities. NeuroSignals 19:128-141. https://doi. org/10.1159/000327819

7. De Rubeis S, He X, Goldberg AP, Poultney CS, Samocha K, Cicek AE, Kou Y, Liu L, Fromer M, Walker S et al (2014) Synaptic, transcriptional and chromatin genes disrupted in autism. Nature 515:209-215. https://doi. org/10.1038/nature13772

8. Feng G, Mellor RH, Bernstein M, Keller-Peck C, Nguyen QT, Wallace M, Nerbonne JM, Lichtman JW, Sanes JR (2000) Imaging neuronal subsets in transgenic mice expressing multiple spectral variants of GFP. Neuron 28:41-51

9. Hu HT, Shih PY, Shih YT, Hsueh YP (2016) The involvement of neuronspecific factors in dendritic spinogenesis: molecular regulation and association with neurological disorders. Neural Plast 2016:5136286. https ://doi.org/10.1155/2016/5136286

10. Huang TN, Chuang HC, Chou WH, Chen CY, Wang HF, Chou SJ, Hsueh YP (2014) Tbr1 haploinsufficiency impairs amygdalar axonal projections and results in cognitive abnormality. Nat Neurosci 17:240-247. https://doi. org/10.1038/nn.3626

11. Iossifov I, Ronemus M, Levy D, Wang Z, Hakker I, Rosenbaum J, Yamrom B, Lee YH, Narzisi G, Leotta A et al (2012) De novo gene disruptions in children on the autistic spectrum. Neuron 74:285-299. https://doi. org/10.1016/..neuron.2012.04.009

12. Lin CW, Hsueh YP (2014) Sarm1, a neuronal inflammatory regulator, controls social interaction, associative memory and cognitive flexibility in mice. Brain Behav Immun 37:142-151. https://doi.org/10.1016/j. bbi.2013.12.002

13. Lo CA, Kays I, Emran F, Lin TJ, Cvetkovska V, Chen BE (2019) Quantification of protein levels in single living cells. Cell Rep 26:3172. https://doi. org/10.1016/j.celrep.2019.02.112

14. Ohoka Y, Takai Y (1998) Isolation and characterization of cortactin isoforms and a novel cortactin-binding protein, CBP90. Genes Cells 3:603-612

15. Pédelacq JD, Cabantous S, Tran T, Terwilliger TC, Waldo GS (2006) Engineering and characterization of a superfolder green fluorescent protein. Nat Biotechnol 24:79-88. https://doi.org/10.1038/nbt1172
16. Rohatgi R, Ho HY, Kirschner MW (2000) Mechanism of N-WASP activation by CDC42 and phosphatidylinositol 4, 5-bisphosphate. J Cell Biol 150:1299-1310. https://doi.org/10.1083/jcb.150.6.1299

17. Ruzzo EK, Perez-Cano L, Jung JY, Wang LK, Kashef-Haghighi D, Hartl C, Singh C, Xu J, Hoekstra JN, Leventhal O et al (2019) Inherited and De Novo genetic risk for autism impacts shared networks. Cell 178:850-866. e826. https://doi.org/10.1016/j.cell.2019.07.015

18. Sanders SJ, He X, Willsey AJ, Ercan-Sencicek AG, Samocha KE, Cicek AE, Murtha MT, Bal VH, Bishop SL, Dong S et al (2015) Insights into Autism spectrum disorder genomic architecture and biology from 71 risk loci. Neuron 87:1215-1233. https://doi.org/10.1016/..neuron.2015.09.016

19. Sandin S, Lichtenstein P, Kuja-Halkola R, Hultman C, Larsson H, Reichenberg A (2017) The heritability of Autism spectrum disorder. JAMA 318:1182-1184. https://doi.org/10.1001/jama.2017.12141

20. Shih PY, Hsieh BY, Lin MH, Huang TN, Tsai CY, Pong WL, Lee SP, Hsueh YP (2020) CTTNBP2 controls synaptic expression of zinc-related autism-associated proteins and regulates synapse formation and autism-like behaviors. Cell Rep 31:107700. https://doi.org/10.1016/j.celrep.2020.107700

21. Shih PY, Lee SP, Chen YK, Hsueh YP (2014) Cortactin-binding protein 2 increases microtubule stability and regulates dendritic arborization. J Cell Sci 127:3521-3534. https://doi.org/10.1242/jcs.149476

22. Wang H, Yang H, Shivalila CS, Dawlaty MM, Cheng AW, Zhang F, Jaenisch $R$ (2013) One-step generation of mice carrying mutations in multiple genes by CRISPR/Cas-mediated genome engineering. Cell 153:910-918. https://doi.org/10.1016/j.cell.2013.04.025

23. Yang $H$, Wang $H$, Jaenisch $R$ (2014) Generating genetically modified mice using CRISPR/Cas-mediated genome engineering. Nat Protoc 9:19561968. https://doi.org/10.1038/nprot.2014.134

\section{Publisher's note}

Springer Nature remains neutral with regard to jurisdictional claims in published maps and institutional affiliations.
Ready to submit your research? Choose BMC and benefit from:

- fast, convenient online submission

- thorough peer review by experienced researchers in your field

- rapid publication on acceptance

- support for research data, including large and complex data types

- gold Open Access which fosters wider collaboration and increased citations

- maximum visibility for your research: over 100M website views per year

At BMC, research is always in progress.

Learn more biomedcentral.com/submissions 